\title{
TSG- 6 secreted by bone marrow mesenchymal stem cells attenuates intervertebral disc degeneration by inhibiting the TLR2/NF-KB signaling pathway
}

\author{
Hao Yang ${ }^{1} \cdot$ Weitian Tian ${ }^{1} \cdot$ Shaocheng Wang ${ }^{2} \cdot$ Xiaohua Liu $^{1} \cdot$ Zhankui Wang $^{1} \cdot$ Lei Hou $^{1} \cdot$ Jiaxi Ge $^{1} \cdot$ Xiao Zhang $^{1} \cdot$ \\ Zhengyu $\mathrm{He}^{3} \cdot$ Xiangrui Wang ${ }^{1}$
}

Received: 9 December 2017 / Revised: 2 January 2018 / Accepted: 16 January 2018

(c) United States \& Canadian Academy of Pathology 2018

\begin{abstract}
Inflammation has been correlated with intervertebral disc degeneration (IDD). Recent evidence suggests that TNF- $\alpha$ stimulated gene 6 protein (TSG-6) secreted by bone marrow mesenchymal stem cells (BMSCs) displays a remarkable ability to inhibit inflammatory processes in a variety of diseases. However, it is unknown whether BMSCs exert their therapeutic effect against IDD by secreting TSG-6. Here we investigated the effects of BMSCs and TSG-6 on IDD and explored the possible underlying mechanisms in vitro and in vivo. We found that BMSCs and TSG-6 reduced the expression of MMP-3 and MMP-13, and increased the expression of collagen II and aggrecan in the IL-1 $\beta$-treated nucleus pulposus cells (NPCs), but the protective effects of BMSCs and TSG- 6 were attenuated when TSG- 6 expression was silenced. We also found that the activation of the TLR2/NF-KB pathway was inhibited by BMSCs and TSG- 6 . The levels of IL- 6 and TNF- $\alpha$ in the degenerated NPCs were reduced and the proliferation of IL-1 $\beta$-treated NPCs was increased in the presence of BMSCs and TSG-6. Furthermore, in vivo experiments showed that BMSCs and TSG-6 restored the MRI T2-weighted signal intensity and increased collagen II and aggrecan expression in the degenerated nucleus pulposus (NP) tissues. Finally, our results showed that BMSCs and TSG-6 downregulated the TLR2/NF-KB signaling and reduced the expression of MMPs and inflammatory cytokines in the degenerated NP tissues. The present study is the first to demonstrate the involvement of TLR2/NF-KB pathway in the potential anti-IDD therapeutic effect of TSG-6, and the results provide new insight into the beneficial effect of BMSCs in the treatment of IDD.
\end{abstract}

\section{Introduction}

Low back pain is one of the leading causes of job-related disability and costs at least $\$ 50$ billion each year in the

\section{Zhengyu He}

zhengyuheshsmu@163.com

$\triangle$ Xiangrui Wang

wangxiangruirjyy@sina.com

1 Department of Anesthesiology, Ren Ji Hospital, School of Medicine, Shanghai Jiao Tong University, Shanghai 200127, China

2 Department of Anesthesiology, Shanghai Tenth People's Hospital, School of Medicine, Tongji University, Shanghai 200072, China

3 Department of Critical Care Medicine, Ren Ji Hospital, School of Medicine, Shanghai Jiao Tong University, Shanghai 200127, China
United States. It is estimated that up to $80 \%$ of adults will experience some form of back pain in their lifetime, and almost $5 \%$ of sufferers become chronically disabled $[1,2]$. Intervertebral disc degeneration (IDD) is a condition characterized by a reduction in the water and proteoglycan (PG) content of the nucleus pulposus (NP) and is one of the dominating contributing factors to low back pain $[3,4]$. The traditional treatments for IDD include nonsurgical and surgical approaches. These methods may alleviate the low back pain but often result in reoccurrence in the same or adjacent discs and fail to eliminate the degeneration of the disc $[5,6]$. Consequently, it is necessary to research more effective treatments for IDD. A normal intervertebral disc forms the main joint between two consecutive vertebrae and is composed of three parts: the highly viscous, cartilaginous $\mathrm{NP}$; annulus fibrosus (AF); and the cartilage endplates. Degenerative changes in NP tissue include decreased cell density, catabolism of the extracellular matrix (ECM), and increased proteinases and inflammatory cytokines [7, 8]. 
Therefore, therapeutic strategies have focused on inhibiting the degeneration of nucleus pulposus cells (NPCs) and their ECM products.

Recently, cell-based therapy, such as bone marrow mesenchymal stem cell (BMSC) treatment, has been developed as a potential strategy for repairing tissue injuries. BMSCs are multi-potent stem cells with relatively low immunogenicity. These cells are easy to isolate from human donors or patients, expand rapidly in culture, and differentiate into several cellular phenotypes in vitro and in vivo. Previous studies have tested the potential of BMSCs to repair tissues by differentiating to replace injured cells. However, recent studies have indicated that transplanted BMSCs exert their therapeutic effect without evidence of long-term engraftment [9]. Therefore, the mechanisms by which BMSCs exert their beneficial effects remain controversial. Currently, an increasing number of studies demonstrate that the protective effects of BMSCs might be due to potent immunomodulatory and immunosuppressive properties via paracrine secretions or cell-to-cell contacts [10-14], and a previous study has found that BMSCs can delay degenerative changes by suppressing inflammation in NPCs [15]. However, the precise mechanisms of this action are not yet clear.

A hallmark of IDD is the significant increase in proinflammatory cytokines, such as interleukin- $1 \beta$ (IL-1 $\beta$ ) and tumor necrosis factor- $\alpha$ (TNF- $\alpha)$, which stimulate the expression of catabolic enzymes, leading to breakdown of the ECM and reduction of the water content of the NP tissue [16-20]. Toll-like receptors (TLRs) are a group of pattern recognition receptors that can mediate innate and adaptive immunity against exogenous or endogenous dangerous ligands and danger-associated molecular patterns produced after tissue injury [21, 22]. TLR activation increases the levels of the catabolic proteases matrix metalloproteinase (MMP)-3 and MMP-13 in disc cells [23, 24]. In particular, TLR2 activates the conserved myeloid differentiation primary response gene 88 (MyD88)-dependent pathway, which results in the activation of nuclear factor (NF)- $\mathrm{kB}$ in the inflammatory pathogenesis of IDD; moreover, several recent studies have found that pro-inflammatory cytokines, such as IL-1 $\beta$, TNF- $\alpha$, HMGB-1, and ECM fragments, such as hyaluronic acid fragments, are endogenous ligands of TLR2 which can strongly activate TLR2 signaling in human intervertebral disc cells [23-25]. Thus, TLR2 may play a crucial role in human disc degeneration $[23,26]$.

$\mathrm{NF}-\kappa \mathrm{B}$ is a central component of the cellular response to damage, stress, and inflammation. Under normal conditions, $\mathrm{NF}-\kappa \mathrm{B}$ is localized primarily in the cytoplasm and maintains an inactive state in association with the inhibitory protein IкB. In response to stimulation, including inflammatory, oxidative, or mechanical stress, IкB is phosphorylated by IкB kinase and is then ubiquitinated and degraded; its degradation allows NF- $\mathrm{KB}$ to translocate into the nucleus where it binds to its cognate DNA site to induce transcription of NF-kB-targeted genes, e.g., TNF- $\alpha$, IL- $1 \beta$, MMP-3, and MMP-13 [27-29]. Numerous studies have shown that activation of the NF- $\mathrm{KB}$ signaling system occurs in IDD, especially in the NP tissue [30-33]. Moreover, previous studies have found that inhibiting the activation of the NF- $\mathrm{kB}$ can delay the process of IDD $[34,35]$.

TNF- $\alpha$-stimulated gene 6 protein (TSG-6) is a $30 \mathrm{kD}$ glycoprotein that can exert an anti-inflammatory effect. Several studies have shown that BMSCs can secrete a variety of anti-inflammatory proteins, such as TSG-6, to modulate the inflammatory microenvironment partly by inhibiting the activation of the TLR2/ NF- KB signaling pathway [36, 37]. These results demonstrate that TSG-6 repairs the injured tissue by attenuating the inflammatory cascade.

To our limited knowledge, there has been no research about the effects of TSG-6 secreted by BMSCs on degeneration of the intervertebral disc. Therefore, in the present study, we examined the anti-inflammatory effects of BMSCs on the process of IDD and investigated whether TSG-6 secreted from BMSCs exerts its protective effect through suppressing the activation of the TLR2/NF- $\mathrm{KB}$ signaling pathway. We conducted our experiments with an IL-1 $\beta$-treated NPC model in vitro and a rat intervertebral disc puncture model in vivo.

\section{Materials and methods}

\section{Ethics statement}

All experiments and surgical procedures were approved by the Animal Care and Use Committee of the Shanghai Jiaotong University School of Medicine, adhered to the recommendations in the Guide for the Care and Use of Laboratory Animals published by the National Institutes of Health, and complied with the relevant sections of the ARRIVE (Animal Research: Reporting of In Vivo Experiments) guidelines.

\section{Animals}

Surgical procedures were performed on adult male Sprague-Dawley (SD) rats weighing 200-250 g. All rats were housed in a standard animal care room with a $12 \mathrm{~h} / 12$ $\mathrm{h}$ light/dark cycle and had free access to food and water.

\section{Isolation, culture, and identification of BMSCs}

SD rat primary BMSCs were prepared as previously described. Briefly, primary BMSCs were obtained from the 
bone marrow of the tibias and femurs of 4-week-old male SD rats under aseptic conditions. A syringe fitted with an 18-gauge needle was inserted into the bone marrow cavity, and then the bone marrow was flushed out with $5 \mathrm{ml}$ of DMEM/F12 (Gibco, Gibco, Grand Island, NY, USA) supplemented with $10 \%$ fetal bovine serum (FBS; Gibco), 100 $\mathrm{U} / \mathrm{ml}$ penicillin, and $100 \mu \mathrm{g} / \mathrm{ml}$ streptomycin (Gibco). The cells were placed in $25 \mathrm{~cm}^{2}$ culture flasks (Corning, NY, USA) and cultured at $37^{\circ} \mathrm{C}$ under $5 \% \quad \mathrm{CO}_{2}$ and $90 \%$ humidity. After $48 \mathrm{~h}$ of incubation, the non-adherent cells were removed and fresh culture medium was added to the flasks. The medium was changed every 3 days. When the cells reached $80-90 \%$ confluence, the adherent cells were washed with phosphate-buffered saline (PBS) and harvested by incubation with $1 \mathrm{ml}$ of $0.25 \%$ trypsin and $1 \mathrm{mM}$ ethylenediaminetetraacetic acid (EDTA) for $2 \mathrm{~min}$ at $37^{\circ} \mathrm{C}$. The trypsin was neutralized with $5 \mathrm{ml}$ of the complete medium and passaged at a dilution of 1:3. BMSCs at passage 3 were used for experiments. BMSCs were assessed using flow cytometry to detect cells that expressed the typical markers; the antibodies were as follows: CD29-PE, CD45-FITC, CD90-FITC, CD49e-FITC, or isotype control (eBioscience, San Diego, CA, USA).

\section{BMSC differentiation assays}

Prior to using BMSCs for in vitro and in vivo experiments, we examined their multilineage differentiation under adipogenic, chondrogenic, and osteogenic differentiation conditions using oil Red O stain (Sigma-Aldrich, St. Louis, MO, USA), Alcian blue stain (Sigma-Aldrich), and Alizarin red stain (Sigma-Aldrich), respectively.

BMSCs were seeded into 6-well plates (Corning) at $1.5 \times 10^{5}$ cells/well, and cultured until reaching confluence. For adipogenic differentiation analysis, cells were incubated in adipogenic induction medium, $\alpha$-minimum essential medium ( $\alpha$-MEM; Gibco) containing 10\% FBS (Gibco), 1 $\mathrm{mmol} / \mathrm{l}$ dexamethasone (Sigma-Aldrich, St. Louis, MO, USA), $0.2 \mathrm{mmol} / \mathrm{l}$ indomethacin (Sigma-Aldrich), $10 \mathrm{mg} / \mathrm{ml}$ insulin(Sigma-Aldrich), and $0.5 \mathrm{mmol} / \mathrm{l}$ 3-isobutyl-1methyl-xanthine (Sigma-Aldrich) for 21 days. The adipogenic induction medium was changed every 3 days. After 21 days of induction, the cells were fixed in PBS containing $10 \%$ formaldehyde solution and stained with oil Red O. For chondrogenic differentiation analysis, cells were treated with chondrogenic induction medium low-glucose Dulbecco's modified Eagle's medium (DMEM; Gibco) supplemented with $2 \mathrm{mg} / \mathrm{l}$ insulin (Sigma-Aldrich), $3 \mathrm{mg} / \mathrm{l}$ transferrin (Sigma-Aldrich), $1 \mathrm{mmol} / \mathrm{l}$ sodium pyruvate (Sigma-Aldrich), $100 \mathrm{nmol} / \mathrm{l}$ dexamethasone (SigmaAldrich), and $10 \mu \mathrm{g} / 1$ transforming growth factor $\beta 1$ (PeproTech, USA). The chondrogenic induction medium was changed every 3 days. After 28 days of induction, the cells were then stained with Alcian blue. For osteogenic differentiation analysis, cells were incubated in $\alpha$-MEM (Gibco) supplemented with 10\% FBS (Gibco), 100 $\mathrm{nmol} / \mathrm{l}$ dexamethasone (Sigma-Aldrich), $10 \mathrm{mmol} / \mathrm{l}$ $\beta$-glycerophosphate (Sigma-Aldrich), and $50 \mu \mathrm{mol} / \mathrm{l}$ L-ascorbic acid 2-phosphate (Sigma-Aldrich). The medium was replaced every 3 days. After 28 days of induction, the mineralized osteocytes were visualized with Alizarin red staining.

\section{Isolation and culture of primary NPCs}

SD rat intervertebral discs were extracted immediately after killing. Under sterile operating conditions, lumbar spinal columns were removed and rinsed with PBS three times. The fiber ring was cut and then the gel-like NP was separated from the $\mathrm{AF}$ under a dissecting microscope. The NP tissue was subsequently treated with $0.25 \%$ type II collagenase for $3 \mathrm{~h}$, and the resulting solution was centrifuged at $1000 \mathrm{rpm}$ for $5 \mathrm{~min}$ to precipitate the cells. The cells were then placed in $25 \mathrm{~cm}^{2}$ culture flasks (Corning) with DMEM/ F12 (Gibco) supplemented with 10\% FBS (Gibco), 100 U/ $\mathrm{ml}$ penicillin, and $100 \mu \mathrm{g} / \mathrm{ml}$ streptomycin (Gibco) and cultured at $37^{\circ} \mathrm{C}$ with $5 \% \mathrm{CO}_{2}$ and saturated humidity. NPCs adhered to the bottom of culture flask after 3 days. The medium was changed every 3 days.

\section{Activation of BMSCs using TNF- $a$ to secrete TSG-6}

BMSCs were activated using TNF- $\alpha$, as described previously [36]. Briefly, BMSCs were plated in 6-well plates at $1 \times 10^{5}$ cells in $2 \mathrm{ml}$ of DMEM/F12 with $10 \%$ FBS per well and were incubated for 1 day. The medium was then changed to DMEM/F12 containing $1 \%$ FBS and $10 \mathrm{ng} / \mathrm{ml}$ TNF- $\alpha$ (R\&D Systems, Minneapolis, MN, USA). After incubation for $18 \mathrm{~h}$, the cells were trypsinized using $0.25 \%$ trypsin with $1 \mathrm{mM}$ EDTA (Gibco) for $2 \mathrm{~min}$ at $37^{\circ} \mathrm{C}$. To confirm the increased expression of TSG-6, we extracted RNA from cell aliquots (RNeasy Mini Kit; QIAGEN) and measured the expression of TSG-6 using real-time reverse transcription-PCR (RT-PCR).

\section{Transfection of BMSCs with TSG-6 siRNA}

BMSCs at passage 3 were used for transfection. Briefly, BMSCs were plated at $1 \times 10^{5}$ cells per well in 6-well dishes and cultured for $24 \mathrm{~h}$. The cells were then transfected with TSG-6 small interfering RNA (siRNA) or negative control siRNA (Genomeditech, Shanghai, China) using Lipofectamine $^{\mathrm{TM}}$ RNAiMAX and transfection medium (OptiMEM I Medium; Gibco) according to the manufacturer's instructions. After $6 \mathrm{~h}$, the medium was replaced with DMEM/F12 containing 10\% FBS. To confirm 
knockdown of TSG-6, we extracted RNA from aliquots of the cells and analyzed for TSG-6 expression using real-time RT-PCR.

\section{Transwell co-culture of NPCs with BMSCs and TSG-6}

A 6-well transwell system $(0.4 \mu \mathrm{m}$ pore size membrane; Corning) was used to assess the effects of BMSCs and TSG- 6 on NPCs that had been stimulated using IL-1 $\beta$. A total of $1 \times 10^{5}$ NPCs were placed in the lower chamber, and 1 day later the NPCs were either treated with $75 \mathrm{ng} / \mathrm{ml}$ of IL-1 $\beta$ for $24 \mathrm{~h}$ or left untreated; subsequently, these NPCs were co-cultured with one of the following treatments in the upper chamber: (1) control medium, (2) $1.0 \times 10^{5}$ TNF- $\alpha$-activated BMSCs, (3) $1.0 \times 10^{5}$ activated BMSCs transfected with TSG-6 siRNA2, (4) $1.0 \times 10^{5}$ activated BMSCs transfected with negative control siRNA, (5) rTSG6 at $1 \mathrm{ng} / \mathrm{ml},(6)$ rTSG-6 at $10 \mathrm{ng} / \mathrm{ml}$, or (7) rTSG-6 at 100 $\mathrm{ng} / \mathrm{ml}$.

To clarify the mechanisms of BMSC-based therapy, we subjected a total of $1 \times 10^{5} \mathrm{NPCs}$ to treatment with $400 \mathrm{ng} /$ $\mathrm{ml}$ of the TLR2 specific agonist Pam3CSK4 (InvivoGen, San Diego, CA, USA) or no treatment for $24 \mathrm{~h}$, and then these NPCs were co-cultured with one of the following treatments in the upper chamber: (1) control medium, ([2) $1.0 \times 10^{5}$ TNF- $\alpha$-activated BMSCs, or (3) rTSG-6 at 100 $\mathrm{ng} / \mathrm{ml}$.

\section{Real-time RT-PCR}

After $24 \mathrm{~h}$ of transwell co-culture, the expression levels of MMP-3, MMP-13, aggrecan, and collagen II mRNA in NPCs were evaluated using real-time RT-PCR. In brief, total RNA was isolated from NPCs using an RNeasy Mini Kit (QIAGEN) according to the manufacturer's instructions. Complementary DNA synthesis was performed using PrimeScript RT Master Mix (Takara, China), and real-time RT-PCR was performed on a LightCycler 480 real-time PCR system (Roche, USA) using iTaq universal SYBR Green Supermix (Bio-Rad, Hercules, CA, USA). $\beta$-Actin was employed as the endogenous control. The primer sequences were designed using Primer 5.0 software and are listed in Table 1. The data are presented as relative $\mathrm{Ct}$ values. The $2^{-\Delta \Delta C t}$ method was employed to calculate relative expression levels.

\section{Western blot analysis}

Total protein of NPCs or NP tissues was extracted using RIPA lysis buffer containing a protease inhibitor, a phosphatase inhibitor, and phenylmethylsulfonyl fluoride (Beyotime, Shanghai, China). Protein concentrations were estimated using a bicinchoninic acid protein assay kit
Table 1 Sequences of primers used in real-time RT-PCR

\begin{tabular}{lll}
\hline mRNA & Primers & Sequences $\left(5^{\prime}-3^{\prime}\right)$ \\
\hline TSG6 & Upstream & AGGCTGTTTGGCTGACTATGT \\
& Downstream & TTTCCTGTGCTGATGATGTCTT \\
Aggrecan & Upstream & CAGATGGCACCCTCCGATAC \\
& Downstream & GACACACCTCGGAAGCAGAA \\
Collagen II & Upstream & CTCAAGTCGCTGAACAACCA \\
& Downstream & GTCTCCGCTCTTCCACTCTG \\
MMP13 & Upstream & CCTATGGTGATGATGATGATGATG \\
& Downstream & CACTGTAGACTTCTTCAGGATTC \\
MMP3 & Upstream & CTCTCAAGATGATGTAGATGGT \\
& Downstream & CTAACATTGGTAAGGTCTCAG \\
\hline
\end{tabular}

(Thermo Scientific, Rockford, IL, USA). The proteins were separated by $10 \%$ sodium dodecyl sulfate-polyacrylamide gel electrophoresis and transferred to a polyvinylidene difluoride membrane (Millipore, Billerica, MA, USA). The membranes were blocked with Tris-buffered saline and TWEEN-20 containing 5\% non-fat dry milk and then incubated overnight at $4{ }^{\circ} \mathrm{C}$ in the presence of primary antibodies against MMP-3 (1:1000 dilution; Abcam, USA), MMP-13 (1:4000 dilution; Abcam, USA), aggrecan (1:1000 dilution; Abcam, USA), collagen II (1:5000 dilution; Abcam, USA), TLR2, MyD88 (1:1000 dilution; Abcam, USA), p65, or phospho-p65 (1:1000 dilution; Cell Signaling Technology, USA). The secondary antibodies were applied for $1 \mathrm{~h}$ at room temperature. The immunoblots were visualized using enhanced chemiluminescence (Thermo Scientific), and relative protein concentrations were measured using Quantity One software (Bio-Rad). The expression level of $\beta$-actin was used as an internal control.

\section{Measurement of IL- 6 and TNF- $a$ by enzyme-linked immunosorbent assay (ELISA)}

The protein levels of IL- 6 and TNF- $\alpha$ in the cell culture supernatant (Abcam, USA) and NP tissue (Sigma-Aldrich, USA) were measured with an ELISA kit according to the manufacturer's instructions.

\section{Immunofluorescence staining}

Immunofluorescence staining was performed as previously described. A total of $1 \times 10^{5}$ NPCs were grown on glass slides placed in the lower chamber, and the NPCs were treated with $75 \mathrm{ng} / \mathrm{ml}$ of $\mathrm{IL}-1 \beta$ for $24 \mathrm{~h}$ or left untreated. Subsequently, these NPCs were co-cultured with one of the following treatments in the upper chamber: (1) control medium, (2) $1.0 \times 10^{5} \mathrm{TNF}-\alpha$-activated MSCs, or (3) rTSG6 at $100 \mathrm{ng} / \mathrm{ml}$. After $24 \mathrm{~h}$ of transwell co-culture, the cells were washed with PBS and fixed with freshly prepared $4 \%$ 
paraformaldehyde for $15 \mathrm{~min}$ at room temperature, then washed three more times with PBS. Each cover slip was then blocked in 5\% BSA and subsequently incubated with anti-NF-кB p65 antibody (1:300 dilution; Cell Signaling Technology, USA) overnight at $4{ }^{\circ} \mathrm{C}$. The next day, all samples were washed with PBS and incubated with Cy3labeled goat anti-rabbit IgG for $1 \mathrm{~h}$ (1:300 Beyotime) at room temperature. After the samples were washed with PBS, 4',6-diamidino-2-phenylindole (Sigma-Aldrich) was used to stain the cell nuclei. The slides were visualized with fluorescence microscopy (Leica, Heidelberg, Germany), and the percentage of NF- $\mathrm{kB}$ p 65 translocation to the nucleus was determined by examining at least 100 cells per slide and analyzing the images with ImageJ version $1.44(\mathrm{NIH}$, USA).

\section{Cell proliferation assay}

To investigate the capacity of BMSCs and TSG-6 to facilitate NPC proliferation, the Cell Counting Kit-8 assay (CCK-8; Dojindo, Kumamoto, Japan) was used. In brief, a total of $1 \times$ $10^{5}$ NPCs were placed in the lower chamber of the 6-well transwell system, and 1 day later the NPCs were either treated with $75 \mathrm{ng} / \mathrm{ml}$ of IL-1 $\beta$ for $24 \mathrm{~h}$ or left untreated; subsequently, these NPCs were co-cultured with one of the following treatments in the upper chamber: (1) control medium, (2) $1.0 \times 10^{5} \mathrm{TNF}-\alpha$-activated BMSCs, or (3) rTSG-6 at $100 \mathrm{ng} / \mathrm{ml}$. After 1, 2, and 3 days of co-culture, NPCs were transferred to $96-$ well plates and seeded at a density of 3000 cells $(100 \mu \mathrm{l})$ per well with six repeats for each group. The cells were incubated at $37{ }^{\circ} \mathrm{C}$ with $5 \% \mathrm{CO}_{2}$ and saturated humidity for $6 \mathrm{~h}, 10 \mu \mathrm{l}$ of CCK-8 reagent was then added to each well and incubated for $2 \mathrm{~h}$ at $37^{\circ} \mathrm{C}$. The optical density (OD) value was measured at $450 \mathrm{~nm}$ using an absorbance microplate reader (Thermo Scientific, Waltham, MA, USA). The culture medium was used as a blank.

\section{Surgical protocol}

For the needle puncture rat model used in the present study, $24 \mathrm{SD}$ rats were randomly divided into four groups: a Control group, a Needle Puncture group, a Needle Puncture+BMSC transplantation group, and a Needle Puncture+TSG-6 injection group. The rats were anesthetized intraperitoneally with sodium pentobarbital $(40 \mathrm{mg} /$ $\mathrm{kg}$ ) under sterile conditions and then placed into a lateral prone position. The anterior surface of a lumbar intervertebral disc (L4-5) was exposed via a posterolateral retroperitoneal approach. The NP tissues of the lumbar intervertebral disc were punctured 2 times to a depth of 3 $\mathrm{mm}$ and held for $5 \mathrm{~s}$ using a 21-gauge needle to induce disc degeneration. After the puncture, the muscle and skin were closed in layers using 4-0 sterile silk sutures, and the rats were kept warm and monitored closely during postsurgical recovery for at least $4 \mathrm{~h}$. At 4 weeks after the initial puncture, either $1 \times 10^{5}$ BMSCs in $100 \mu \mathrm{l}$ PBS or $100 \mu \mathrm{rTSG}-6(1 \mathrm{ng} / \mathrm{ml}, 10 \mathrm{ng} / \mathrm{ml}, 100 \mathrm{ng} / \mathrm{ml})$ were injected into the rat NP from the contralateral side using a microsyringe with a 21 -gauge needle.

\section{Magnetic resonance imaging (MRI) analysis}

At 4 weeks after the initial puncture, either $1 \times 10^{5}$ BMSCs in $100 \mu \mathrm{l} \mathrm{PBS}$ or $100 \mu \mathrm{lTSG}-6(100 \mathrm{ng} / \mathrm{ml})$ were injected into the rat NP from the contralateral side using a microsyringe with a 21-gauge needle. MRI was performed on each rat at 4 weeks after BMSC and TSG-6 injection to assess the degree of disc degeneration. Midsagittal T2weighted images were obtained from the targeted lumbar discs (L4-5) using a 3.0-Tesla Siemens Trio Tim system (Siemens, Erlangen, Germany).

\section{Histological analysis and immunohistochemistry}

The rat intervertebral discs were harvested at 4 weeks after BMSC and TSG-6 injection. The specimens were fixed in $4 \%$ paraformaldehyde, decalcified in $0.05 \%$ EDTA for 4 weeks, and then embedded in paraffin. Serial sagittal sections of discs ( $5 \mu \mathrm{m}$ thick) were obtained to prepare slides. These sections were stained with hematoxylin and eosin (HE) and Safranin O/Fast Green to assess the disc degeneration. For immunohistochemical detection, sections were incubated with primary antibodies specific to collagen II (1:100 dilution; Abcam, USA) and aggrecan (1:100 dilution; Abcam, USA) overnight at $4{ }^{\circ} \mathrm{C}$. The next day, all sections were washed with PBS and were incubated with biotinylated goat anti-rabbit $\operatorname{IgG}$ at $4{ }^{\circ} \mathrm{C}$ for 60 min and then with streptavidin horseradish peroxidase at $37^{\circ} \mathrm{C}$ for $10 \mathrm{~min}$, followed by color development with diaminobenzidine tetrahydrochloride (Millipore, Billerica, USA). The sections were counterstained with hematoxylin and mounted. The stained sections were examined with an inverted microscope (Leica). All immunohistochemical staining was performed following standard histochemical protocols.

\section{Statistical analyses}

All experiments were performed at least three times, and the data are expressed as the mean value \pm SD. Statistical analyses were performed using SPSS 17.0 software (SPSS, Chicago, IL, USA). One-way analysis of variance was used for comparisons among three or more treatment groups. Student's $t$-test (two tailed) was used for comparisons between two groups. Significance was set at a level of $P<0.05$. 


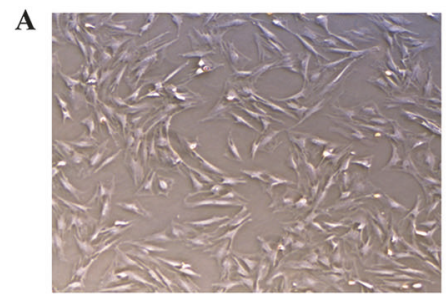

$\mathbf{B}$

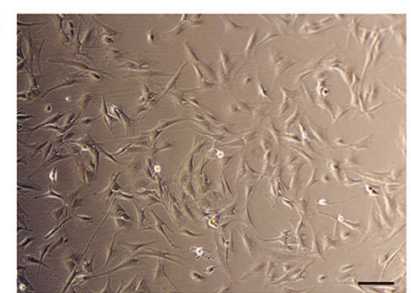

C

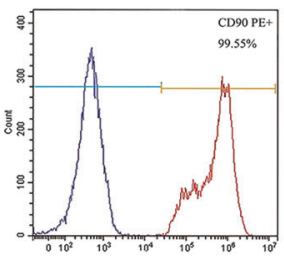

D

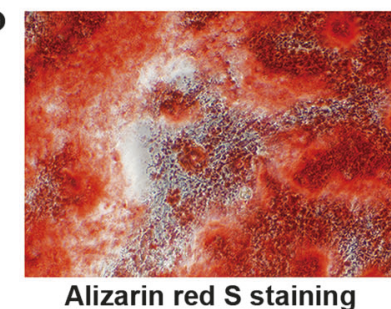

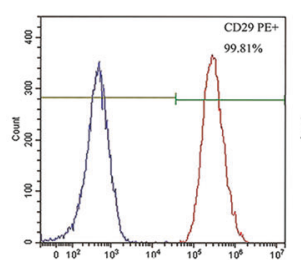
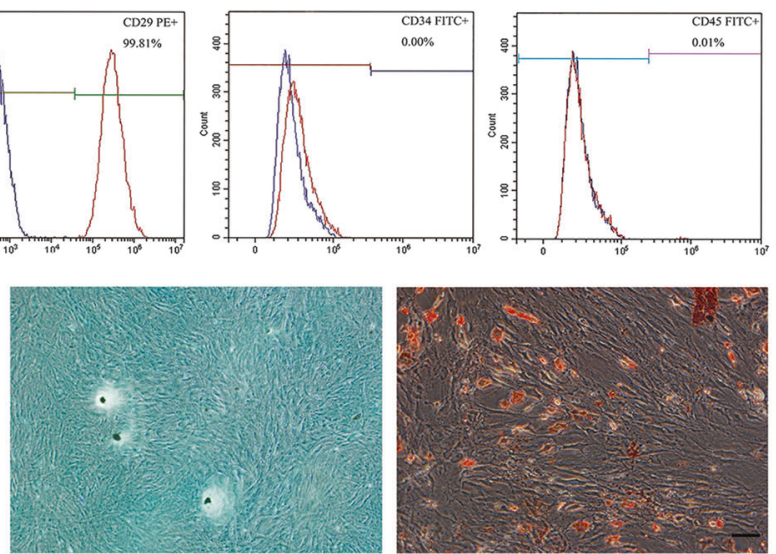

Alcian blue staining

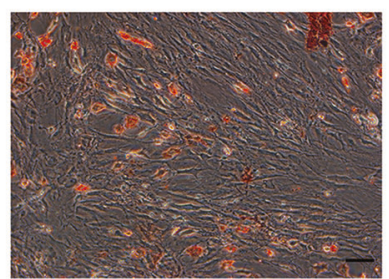

oil Red O staining
Fig. 1 Characterization of BMSCs and NPCs. Morphological observation of $\mathbf{a}$ BMSCs and $\mathbf{b}$ NPCs used in this study. BMSCs were long spindle in shape and were arranged in an organized fashion, and NPCs were mainly triangular, spindle, or polygonal in shape. Scale bar, 100 $\mu \mathrm{m}$. c Flow cytometric analysis of BMSC surface markers. Note that the cells were positive for CD90 and CD29, and negative for CD34 and CD45. The red line represents the target antibody, and the blue line represents the isotype control antibody. $\mathbf{d}$ The multidifferentiation potential of BMSCs in vitro. Alizarin red S staining was used to evaluate osteogenic differentiation, Alcian blue staining was used to evaluate chondrogenic differentiation, and oil Red $\mathrm{O}$ staining was used to evaluate adipogenic differentiation. Scale bar, $100 \mu \mathrm{m}$

\section{Results}

\section{Characterization of BMSCs and NPCs}

Primary cultured BMSCs adhered to the culture dish at approximately $24-48 \mathrm{~h}$ and reached $80-90 \%$ confluence within 6-7 days. BMSCs displayed similar fibroblastic morphologies, and NPCs were mainly oval, spindle, or polygonal in shape (Fig. 1a, b). We used flow cytometric analysis to examine a series of cell surface antigens on the BMSCs at passage 3. Flow cytometric analysis confirmed that the cells were positive for the mesenchymal stem cell markers CD29 and CD90, and negative for the hematopoietic markers CD45 and CD34 (Fig. 1c). In addition, the in vitro differentiation model revealed that the BMSCs successfully differentiated into osteogenic, chondrogenic, or adipogenic lineages under specific differentiation induction (Fig. 1d).

\section{IL-1 $\beta$ induced expression of MMP-3 and MMP-13 and degeneration of NPCs}

Next, we examined the effects of IL- $1 \beta$ on the mRNA and protein expression of MMP-3, MMP-13, collagen II, and aggrecan in rat NPCs. NPCs were treated with $75 \mathrm{ng} / \mathrm{ml}$ IL-1 $\beta$ for $6 \mathrm{~h}, 12 \mathrm{~h}, 24 \mathrm{~h}$ and $48 \mathrm{~h}$. The mRNA and protein expression levels of MMP-3, MMP-13, aggrecan, and collagen II were determined by real-time RT-PCR and western blotting. As shown in Fig. 2a-j, the mRNA and protein expressions of MMP-3 and MMP-13 were significantly upregulated in IL-1 $\beta$-treated NPCs in a time-dependent manner compared with the control group. Conversely,
IL-1 $\beta$ decreased the mRNA and protein expression of aggrecan and collagen II in a time-dependent manner compared with the control group.

\section{BMSCs and TSG- 6 ameliorated IL-1 $\beta$-induced NPC degeneration}

First, we detected whether the mRNA expression of the anti-inflammatory protein TSG-6 was increased in BMSCs after treatment with the inflammatory cytokine TNF- $\alpha$. The data showed that the mRNA expression of TSG-6 was increased in BMSCs after stimulation with TNF- $\alpha$ compared with the control group (Fig. 3a). To explore the effects of BMSCs and TSG-6 on the mRNA expression of MMP-3, MMP-13, aggrecan, and collagen II in IL-1 $\beta$ treated NPCs, we performed co-culture experiments. IL-1 $\beta$ treated NPCs were co-cultured with BMSCs or TSG-6 at different concentrations for $24 \mathrm{~h}$. As expected, BMSCs inhibited the mRNA expression of MMP-3 and MMP-13 in IL-1 $\beta$-treated NPCs. In contrast, the mRNA expression of aggrecan and collagen II was increased significantly when IL-1 $\beta$-treated NPCs were co-cultured with BMSCs (Fig. 3c-f). In addition, we added recombinant TSG-6 to the NPC culture medium during IL- $1 \beta$ treatment. The results showed that the mRNA expression of aggrecan and collagen II increased while that of MMP-3 and MMP-13 reduced dramatically when IL- $1 \beta$-treated NPCs were cultured with $100 \mathrm{ng} / \mathrm{ml}$ TSG-6; this outcome was similar to the results from the BMSC co-cultured group (Fig. 3c-f). To confirm that TSG-6 plays a key role in ameliorating IL-1 $\beta$-induced NPC degeneration, we used TSG- 6 siRNA to knock down the expression of the TSG-6 gene (Fig. 3b). 


\section{A}

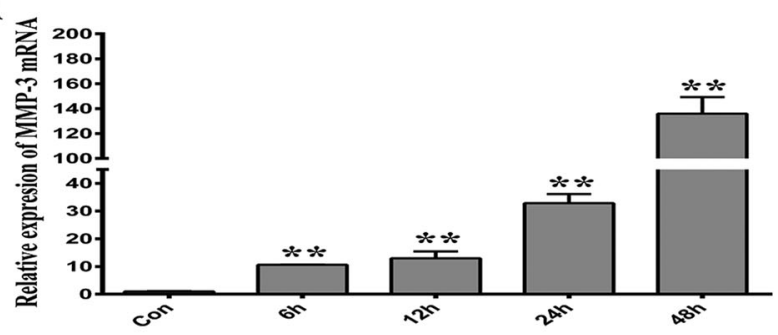

C

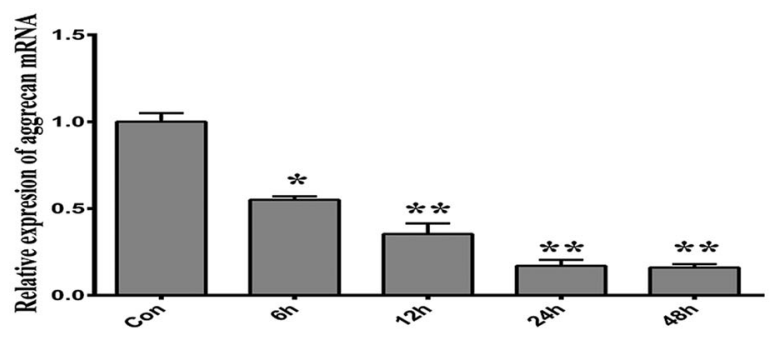

$\mathbf{E}$

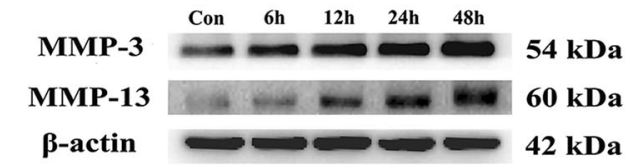

H

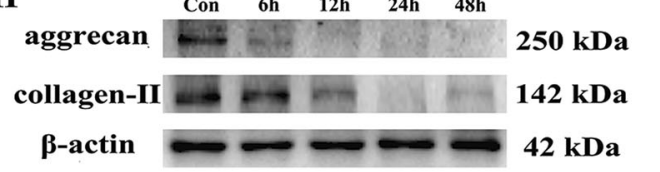

F

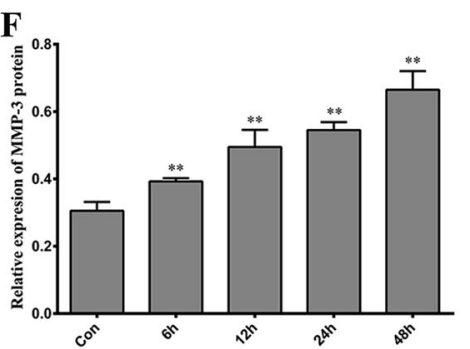

I

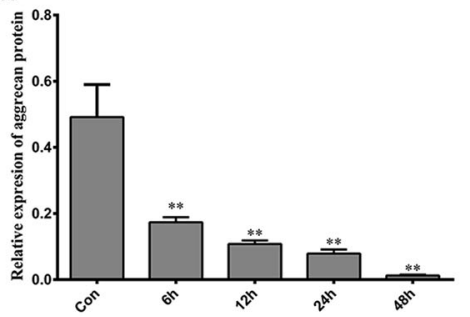

B

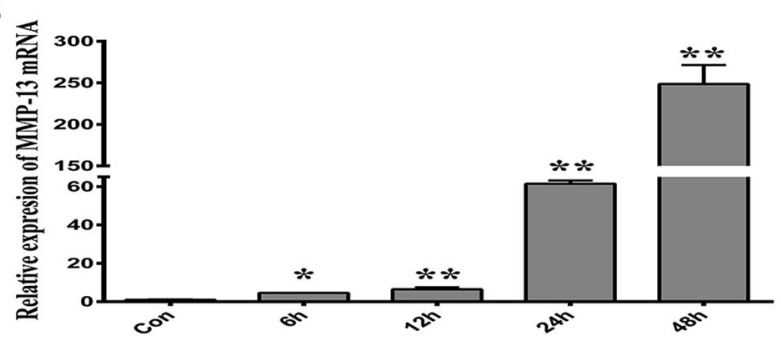

D

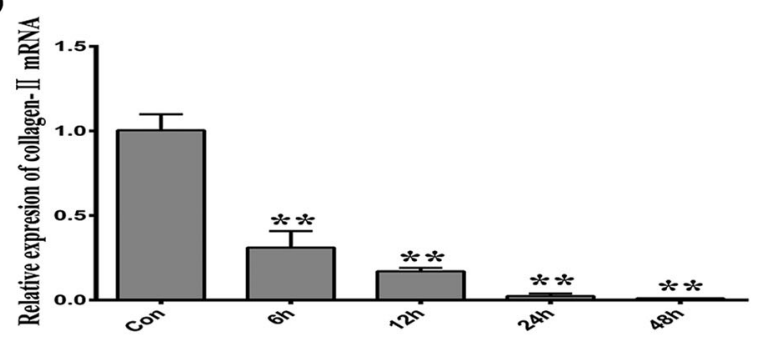

$\mathbf{J}$

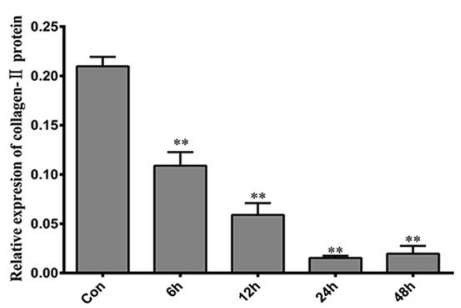

Fig. 2 The mRNA and protein expression of MMP-3, MMP-13, aggrecan, and collagen II in IL- $1 \beta$-treated NPCs. Real-time RT-PCR showed that IL-1 $\beta$ significantly increased the expression of a MMP-3 and $\mathbf{b}$ MMP-13 in NPCs at each time point. In contrast, the expression levels of $\mathbf{c}$ aggrecan and $\mathbf{d}$ collagen II in NPCs were reduced at each time point after IL-1 $\beta$ treatment. All mRNA levels were normalized to the level of $\beta$-actin mRNA. e, $\mathbf{h}$ Representative image of protein levels of MMP-3, MMP-13, aggrecan, and collagen II. Quantitative analysis of western blotting results showed that IL-1 $\beta$ significantly increased the expression of $\mathbf{f}$ MMP-3, $\mathbf{g}$ MMP-13 and decreased the expression levels of $\mathbf{i}$ aggrecan and $\mathbf{j}$ collagen II in NPCs at each time point. The data are expressed as the means $\pm \mathrm{SD}\left(n=6\right.$ in each group). ${ }^{*} P<0.05$; ${ }^{* *} P<0.01$ versus the Control group (Con)

In addition, it has been demonstrated that the antiinflammatory protein TSG-6 secreted by BMSCs can inhibit the activation of the TLR2/NF- $\mathrm{kB}$ signaling pathway [36]. Thus, we examined the effects of BMSCs and TSG-6 on the TLR2/NF- $\mathrm{KB}$ signaling pathway in IL-1 $\beta$-treated NPCs. First, we analyzed NF- $\mathrm{BB}$ p65 nuclear translocation in NPCs using immunofluorescence staining. In the control group, NF-kB p65 largely remained in the cytoplasm. After IL-1 $\beta$ treatment, NF- $\mathrm{kB}$ p65 translocated to the nucleus in NPCs, but to a lesser extent in the BMSC co-culture and TSG-6 treatment groups (Fig. 4a, b).
Recently, the TLR2/NF- $\kappa \mathrm{B}$ signaling pathway has been shown to play an important role in IDD, and inhibiting its activation may slow the degenerative progress [24, 38, 39]. 
Next, we evaluated TLR2, MyD88, phosphorylation of

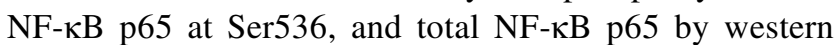
blotting. As expected, compared with the basal protein levels of TLR2, MyD88, and p-p65 in the control group, the protein levels of TLR2, MyD88, and p-p65 were upregulated in IL- $1 \beta$-treated NPCs; in contrast, the IL-1 $\beta$ -
A

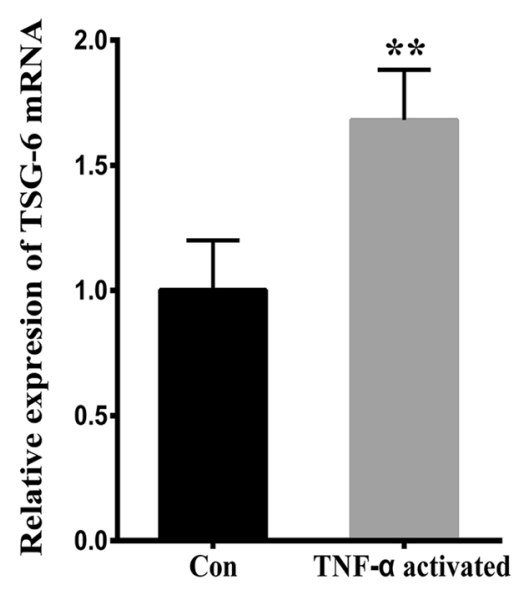

C
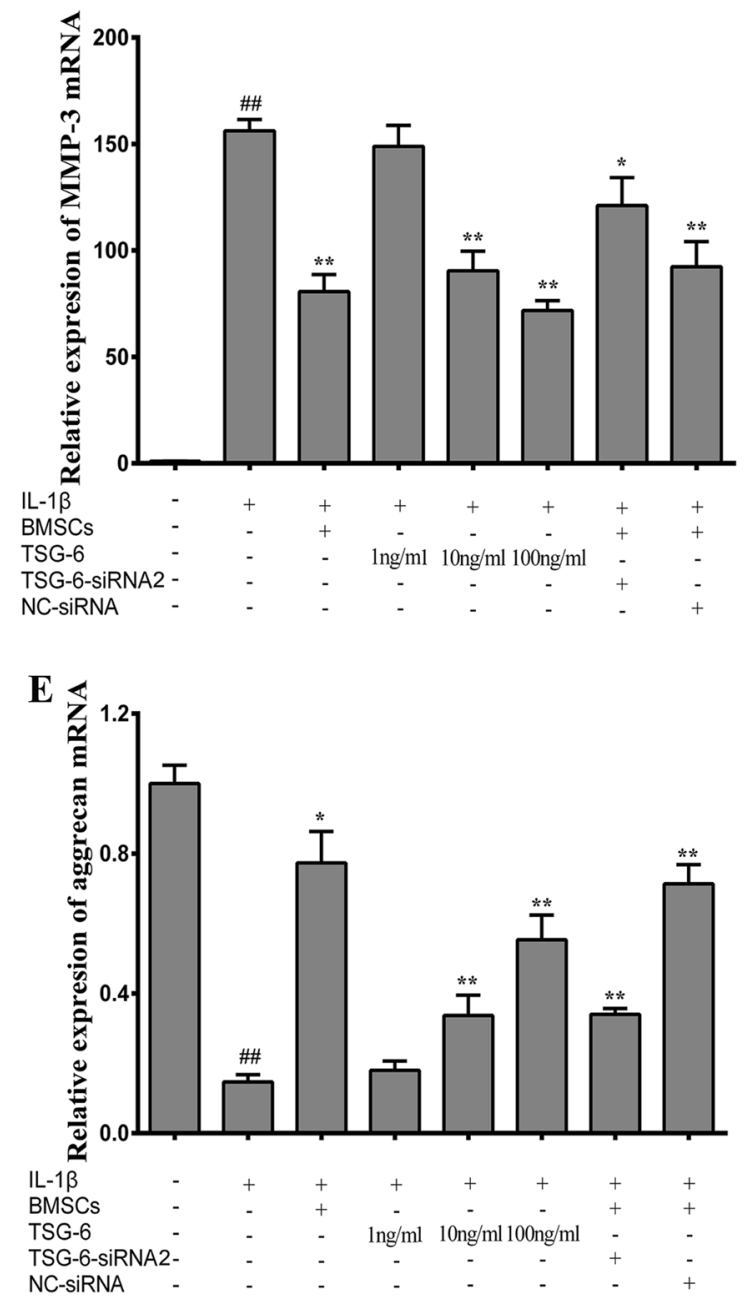

B

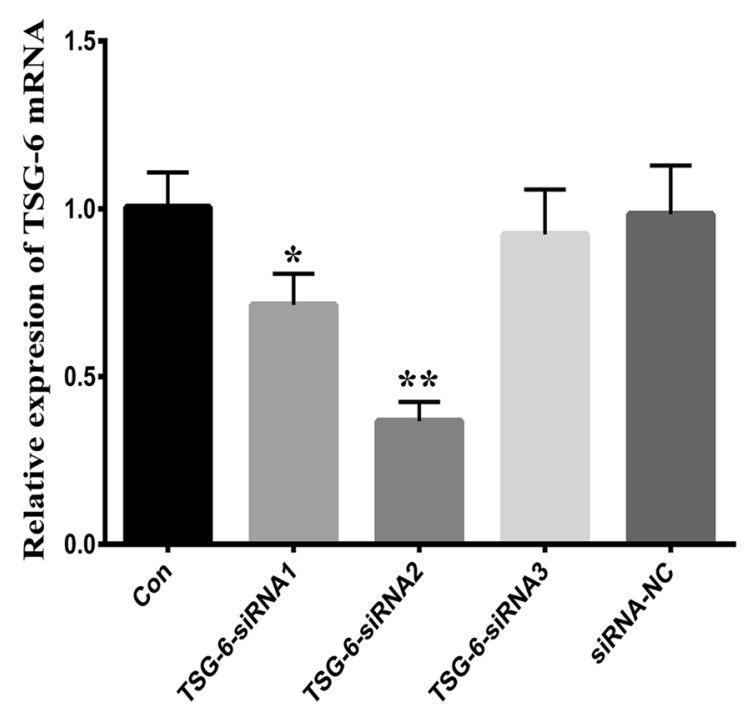

D
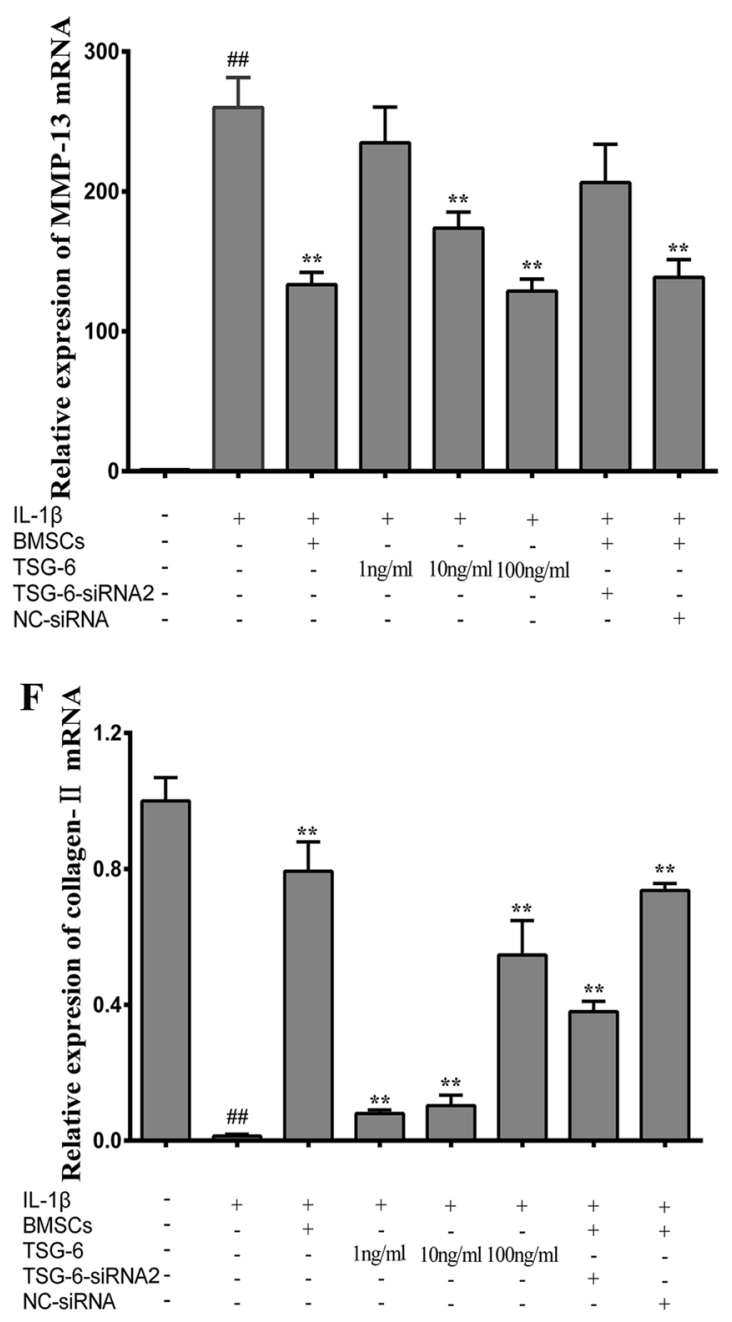
Fig. 3 BMSCs and TSG-6 ameliorated IL-1 $\beta$-induced NPCs Degeneration. a TSG- 6 was increased in BMSCs after stimulation by TNF- $\alpha$. b The gene expression of TSG-6 in control BMSCs, and in BMSCs transfected with TSG-6-siRNA1, TSG-6-siRNA2, TSG-6siRNA3, and negative control siRNA. NPCs were co-cultured for 24 $\mathrm{h}$ with control medium, TNF- $\alpha$-activated BMSCs, and different concentrations of TSG-6 $(1 \mathrm{ng} / \mathrm{ml}, 10 \mathrm{ng} / \mathrm{ml}, 100 \mathrm{ng} / \mathrm{ml})$, TNF- $\alpha-$ activated BMSCs transfected with TSG-6 siRNA2, or TNF- $\alpha$ activated BMSCs transfected with negative control siRNA. c-f The mRNA levels of MMP-3, MMP-13, aggrecan, and collagen II were measured by real-time RT-PCR. All mRNA expression levels were normalized to the level of $\beta$-actin mRNA. The data are expressed as the means $\pm \mathrm{SD}$ ( $n=6$ in each group). ${ }^{\# \#} P<0.01$ versus the normal NPCs group (Con). ${ }^{*} P<0.05 ;{ }^{* *} P<0.01$ versus the IL- $1 \beta$-treated NPC group

induced upregulation of TLR2, MyD88, and p-p65 in NPCs was inhibited after co-culture with BMSCs or treatment with TSG-6 (Fig. 4c-f, h). However, no apparent change in p65 protein level was observed between different groups (Fig. 4c, g). To further determine whether BMSCs and TSG-6 exert the protective effect by suppressing the TLR2/NF- $\mathrm{KB}$ signaling pathway in degenerated NPCs, we explored the effects of BMSCs and TSG-6 on the TLR2/NF- $\kappa$ B signaling pathway in NPCs treated with the specific TLR2 agonist Pam3CSK4. As shown in Fig. 4, Pam3CSK4 significantly increased the protein levels of TLR2, MyD88, and p-p65 in NPCs compared with the control group, whereas the Pam3CSK4-induced upregulation of TLR2, MyD88, and p-p65 in NPCs was inhibited after co-culture with BMSCs or treatment with TSG-6 (Fig. 4i-1, n). However, no obvious change in $\mathrm{p} 65$ expression was observed between groups (Fig. 4i, m).

\section{BMSCs and TSG-6 inhibited the release of inflammatory cytokines by NPCs}

We then examined the effects of BMSCs and TSG-6 on the release of inflammatory cytokines by NPCs. As indicated in Fig. 5a, c, after co-culture with BMSCs or treatment with TSG-6, there were significant reductions in the supernatant levels of IL- 6 and TNF- $\alpha$ in IL-1 $\beta$-treated NPCs compared with the control group. Furthermore, BMSCs and TSG-6 significantly inhibited IL-6 and TNF- $\alpha$ secretion in NPCs induced by Pam3CSK4 (Fig. 5b, d).

\section{BMSCs and TSG- 6 promoted degenerated NPC proliferation}

We examined the effects of BMSCs and TSG-6 on the proliferation of degenerated NPCs. The results showed that IL- $1 \beta$ significantly decreased the proliferation of NPCs compared with the control group. In contrast, after coculture with BMSCs or treatment with TSG-6, the proliferation of IL-1 $\beta$-treated NPCs significantly increased at all time points (Fig. 5e).

\section{BMSCs and TSG- 6 reduced disc degeneration in a rat model}

To explore the therapeutic properties of BMSCs and TSG-6 in vivo, a rat model of BMSC transplantation was established. The NP tissue of the rat lumbar intervertebral disc was punctured using a 21-gauge needle to induce disc degeneration. At 4 weeks after the initial puncture, either $1 \times 10^{5}$ BMSCs or $100 \mu \mathrm{l} \mathrm{TSG}-6$ at different concentrations was injected into the rat NP tissue. To examine the morphological changes in the rat lumbar intervertebral discs from different groups, HE and Safranin O/Fast Green staining were performed. At 4 weeks after BMSC or TSG-6 injection, the HE staining showed that lumbar discs in the control group displayed an intact annulus fibrosus (AF) with a normal pattern of fibrocartilage lamellae. A clear boundary could be seen between the NP and AF, and no cracks existed in the NP. In the surgery group, the degenerative changes of the lumbar intervertebral discs were obvious: the boundary between the NP and AF became indistinct, there was a decrease in the number of nuclear cells in the NP, and cracks existed in the NP and AF. No significant differences were detected between the $1 \mathrm{ng} / \mathrm{ml}$ TSG- 6 injection and surgery groups, and in the $10 \mathrm{ng} / \mathrm{ml}$ TSG-6 injection group, the NP and AF structures were better maintained compared with the surgery group and the $1 \mathrm{ng} / \mathrm{ml}$ TSG- 6 injection group. However, in the BMSC transplantation group and the $100 \mathrm{ng} / \mathrm{ml}$ TSG-6 injection group, most of the NP contents were well preserved: the NP consisted of numerous nuclear cells, the AF was arranged regularly, and the boundary between the NP and AF was minimally interrupted (Fig. 6a). The Safranin O/Fast Green staining also produced intense staining in the BMSC transplantation group and the $100 \mathrm{ng} / \mathrm{ml}$ TSG- 6 injection group, while the staining in the surgery group and the $1 \mathrm{ng} / \mathrm{ml}$ TSG-6 injection group was less than that in the control group (Fig. 6b). The Safranin O/Fast Green staining suggested that BMSC transplantation and TSG-6 $(100 \mathrm{ng} / \mathrm{ml})$ injection significantly reduced the loss of PG in the degenerated intervertebral disc. Above results displayed that TSG-6 alleviated the degenerative changes of the lumbar intervertebral discs in a concentration-dependent manner.

Midsagittal T2-weighted images were obtained from the targeted lumbar discs (L4-5) 4 weeks after injection. The MRI T2-weighted signal intensities in the surgery group were significantly lower than that those in the control group 4 weeks after injection. In contrast, the MRI T2-weighted signal intensities in the BMSC transplantation group and the TSG-6 (100 ng/ml) injection group were significantly higher than in the surgery group 4 weeks after injection (Fig. 6c). 
Fig. 4 BMSCs and TSG-6 suppressed the activation of the TLR2/NF- $\mathrm{KB}$ signaling pathway in degenerated NPCs. a Typical micrographs of immunocytochemical staining are shown for the cytoplasmic and nuclear distribution of NFкB p65. Scale bar: $200 \mu \mathrm{m} ; 75$ $\mu \mathrm{m}$ (insets). b The percentages of cells with NF-кB p65 localized in the nucleus. The NPCs were treated with IL- $1 \beta$ for $24 \mathrm{~h}$ or left untreated, and then these NPCs were cocultured with control medium, activated BMSCs, or TSG-6 $(100 \mathrm{ng} / \mathrm{ml})$ for $24 \mathrm{~h}$. c Representative image of protein levels of TLR2, MyD88, p-p65 and p65. d-h Quantitative analysis of western blotting results showed that BMSCs and TSG-6 significantly inhibited the activation of the TLR2/NF- $\kappa B$ signaling pathway induced by IL- $1 \beta$ in NPCs. The NPCs were treated with the specific TLR2 agonist Pam3CSK4 for $24 \mathrm{~h}$ or left untreated, and then these NPCs were co-cultured with control medium, activated BMSCs, or TSG-6 $(100 \mathrm{ng} / \mathrm{ml})$ for $24 \mathrm{~h}$. i Representative image of protein levels of TLR2, MyD88, p-p65, and p65. j-n Quantitative analysis of western blotting results showed that BMSCs and TSG-6 significantly inhibited the activation of the TLR2/NF- $\kappa \mathrm{B}$ signaling pathway induced by Pam3CSK4 in NPCs. The data are expressed as the means $\pm \mathrm{SD}(n=6$ in each group). ${ }^{\# \#} P<0.01$ versus the control group. ${ }^{* *} P<0.01$ versus the IL-1 $\beta$ or Pam3CSK4-treated NPC group
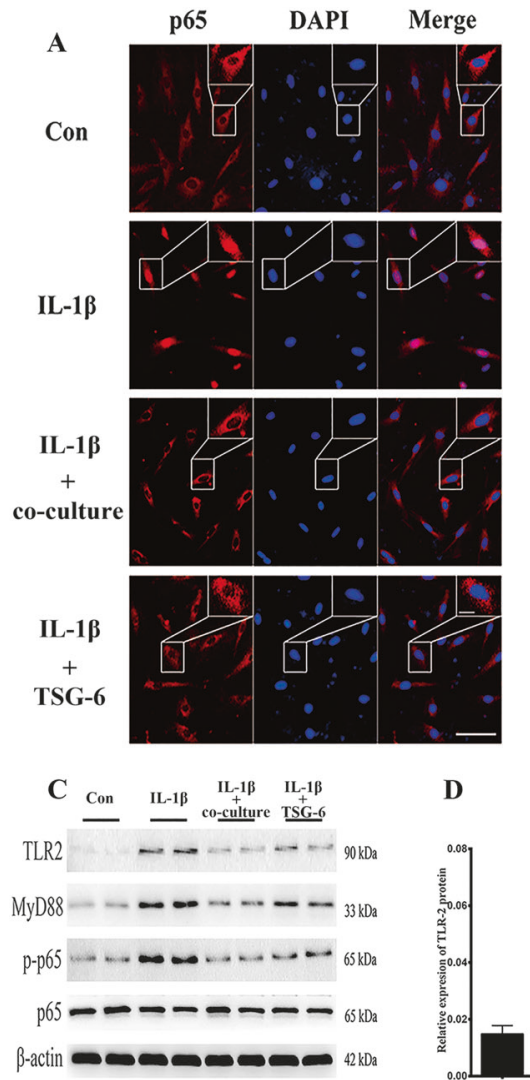

D
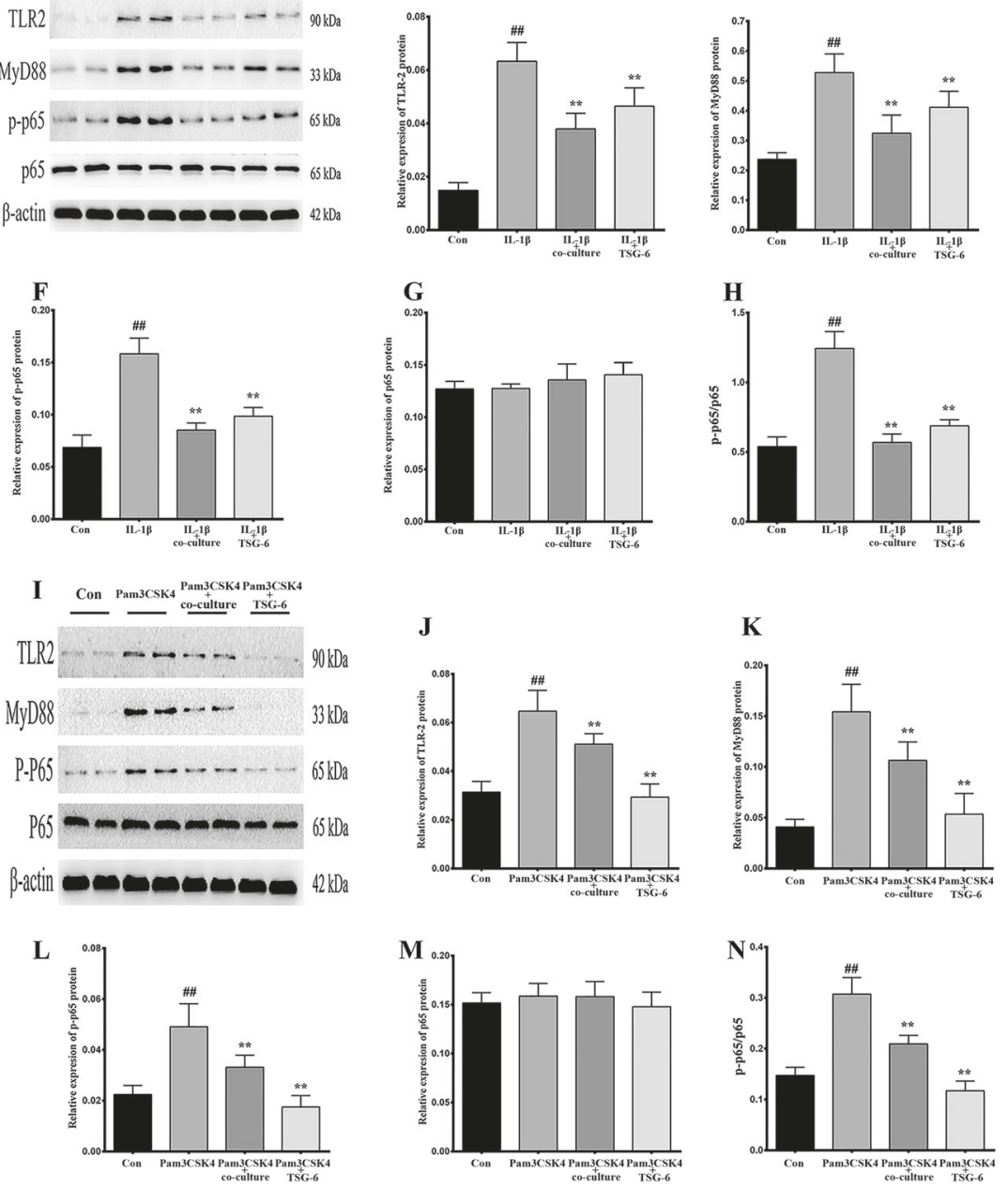
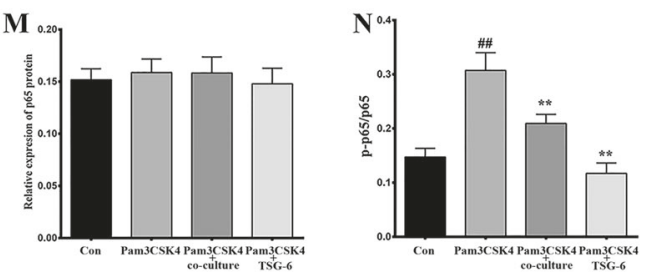

Collagen II and aggrecan are two important components of the ECM in NP tissue and play crucial roles in maintaining the physiological function of the disc; [40, 41] the loss of ECM plays a key role in the development of IDD [42, 43]. Thus, we measured the protein expression of collagen II and aggrecan in NP tissue by 

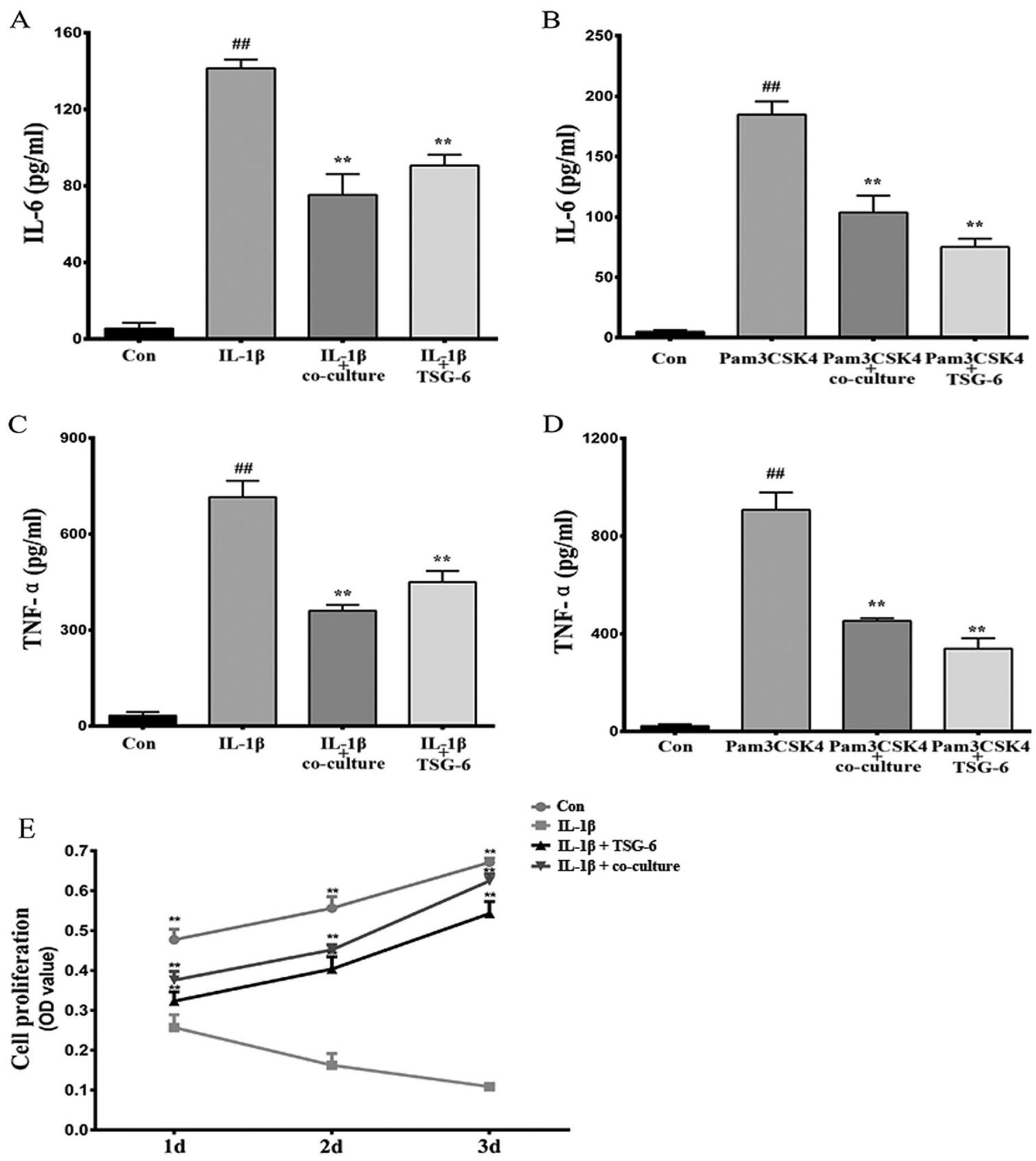

Fig. 5 The effects of BMSCs and TSG- 6 on the release of inflammatory cytokines and the proliferation of degenerated NPCs. The levels of IL- 6 and TNF- $\alpha$ in the cell culture supernatant were measured by ELISA kit. BMSCs and TSG- 6 inhibited the elevated protein levels of a IL- 6 and $\mathbf{c}$ TNF- $\alpha$ induced by IL- $1 \beta$ in NPCs. Similarly, BMSCs and TSG- 6 inhibited the elevated protein levels of $\mathbf{b}$ IL- 6 and $\mathbf{d}$ TNF- $\alpha$

immunohistochemical staining. The results showed that the protein expression of collagen II and aggrecan dramatically reduced in the surgery group compared with the control group; however, BMSC transplantation and TSG-6 injection significantly enhanced the protein levels of collagen II and aggrecan compared with those in the surgery group (Fig. 6d-g).

induced by Pam3CSK4 in NPCs. e Cell proliferation was analyzed using CCK-8. The proliferation of IL-1 $\beta$-treated NPCs significantly increased when co-cultured with BMSCs or treated with TSG-6. The data are expressed as the means $\pm \mathrm{SD}\left(n=6\right.$ in each group). ${ }^{\# \#} P<0.01$ versus the control group. ${ }^{* *} P<0.01$ versus the IL- $1 \beta$ or Pam3CSK4treated NPC group

\section{BMSCs and TSG-6 downregulated the TLR2/NF-KB signaling pathway and reduced the expression of MMPs and inflammatory cytokines in degenerated NP tissues}

To further confirm BMSCs and TSG-6 attenuate intervertebral disc degeneration by inhibiting the TLR2/ NF- $\mathrm{KB}$ signaling pathway in vivo, we examined the protein 
expression levels of TLR2, MyD88, phosphorylation of

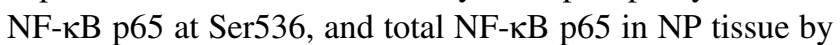
western blotting. The results showed that the protein levels of TLR2, MyD88, and p-p65 in NP tissue of the surgery group were significantly higher than those of the control group, however, at 4 weeks after BMSC transplantation or
A
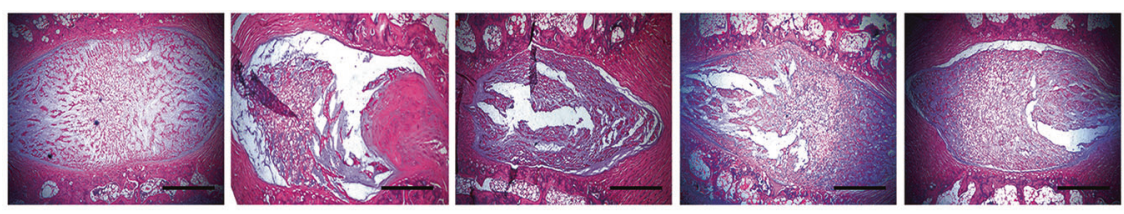

B

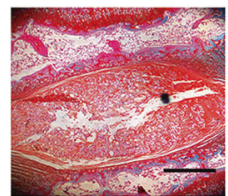

Con

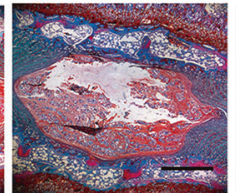

Surgery

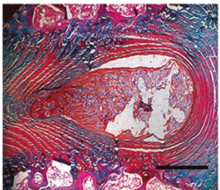

Surgery

Surgery

$+(10)$

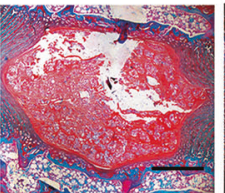

Surgery

$\stackrel{+}{\stackrel{+}{\text { TSG-6(10ng/ml) }})}$

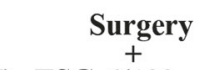

Surgery

TSG-6(100ng/ml)

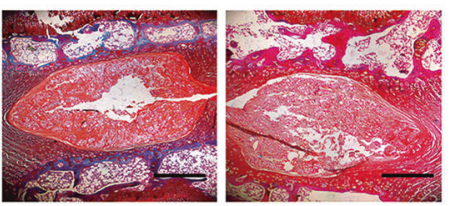

Surgery

BMSCs
C

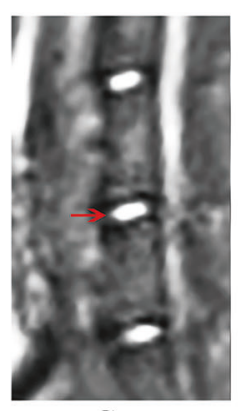

Con

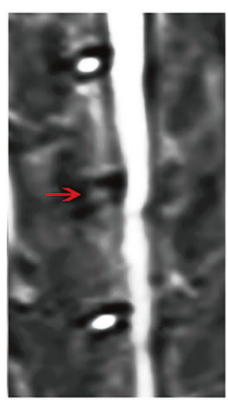

Surgery

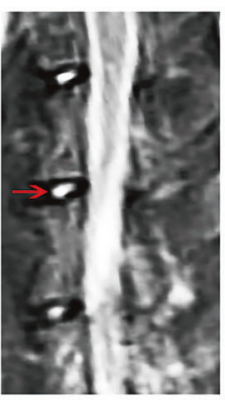

Surgery $\stackrel{+}{\text { BMSCs }}$

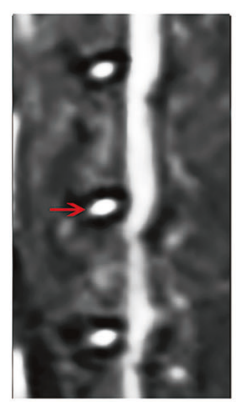

Surgery TSG $\stackrel{+}{-6}$

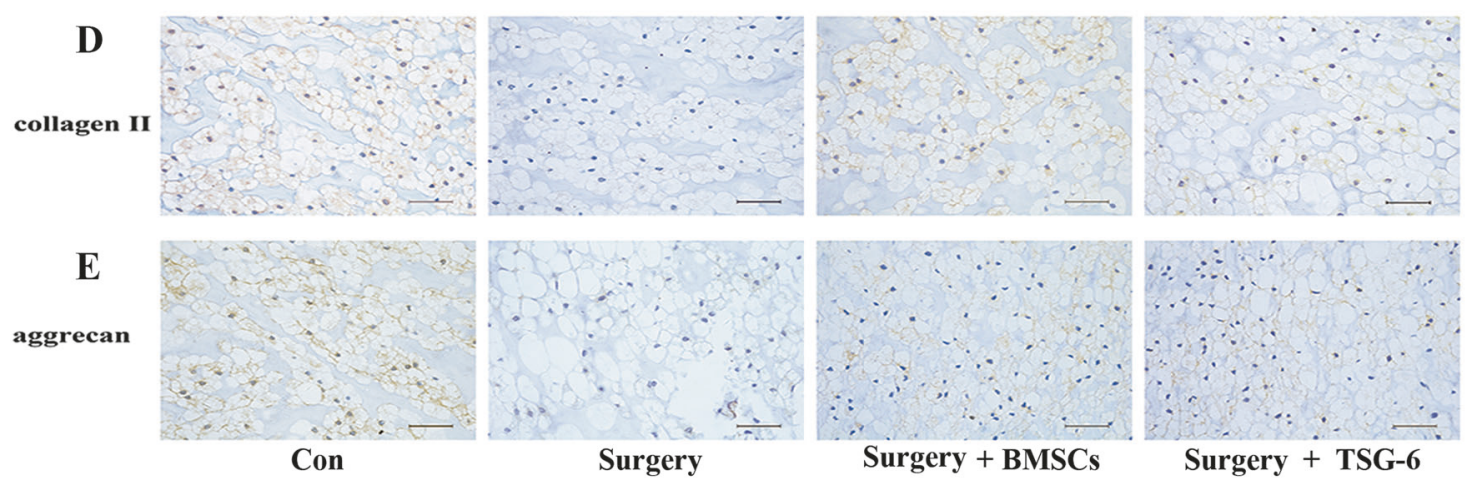

F

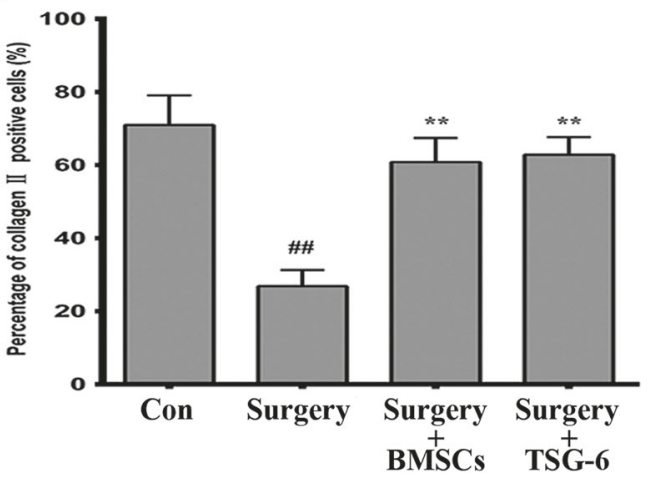

G

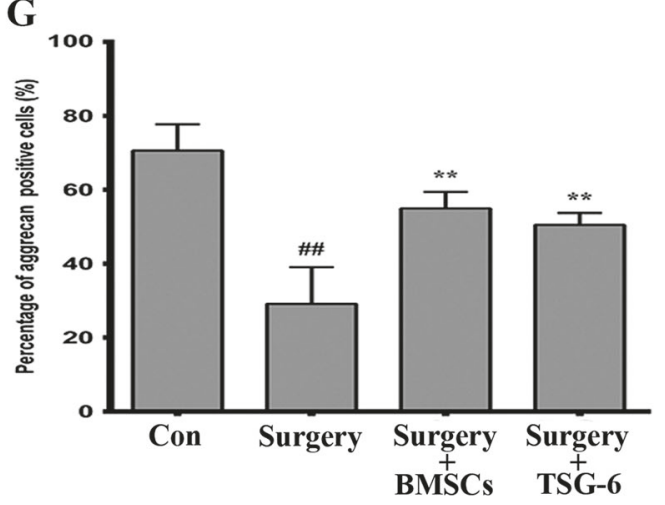


Fig. 6 BMSCs and TSG- 6 ameliorated rat IDD and increased the expression of collagen II and aggrecan in intervertebral discs. Histological analysis of intervertebral discs by a $\mathrm{HE}$ and b Safranin O/Fast Green staining. Scale bar, $400 \mu \mathrm{m}$. c Midsagittal T2-weighted images at 4 weeks after BMSC transplantation and TSG-6 injection into the NP tissue. Representative photomicrographs of immunohistochemical staining of $\mathbf{d}$ collagen II and $\mathbf{e}$ aggrecan in intervertebral discs. The percentages of $\mathbf{f}$ collagen II- and $\mathbf{g}$ aggrecan-positive cells in intervertebral discs. The data are expressed as the means \pm SD $(n=6$ in each group). Scale bar, $100 \mu \mathrm{m}$. ${ }^{\# \#} P<0.01$ versus the control group. ${ }^{* *} P<0.01$ versus the surgery group

TSG-6 injection, the protein levels of TLR2, MyD88, and p-p65 in NP tissue were significantly lower than that for surgery alone (Fig. 7a, c-e). Furthermore, there was no significant difference in 655 protein expression level among the control, surgery, BMSC, and TSG-6 groups (Fig. 7a, f). Next, we observed the protein levels of matrix-degrading enzymes (MMP-3 and MMP-13) in NP tissue among different groups by western blotting. In the surgery group, we found that the protein expression of MMP-3 and MMP-13 in NP tissue was upregulated compared with the control group. However, at 4 weeks after BMSC transplantation or TSG-6 injection, the protein levels of MMP-3 and MMP-13 in NP tissue were dramatically decreased compared with the surgery alone group (Fig. 7b, g, h).

Finally, we measured the protein expression of IL-6 and TNF- $\alpha$ in NP tissue by ELISA. The results showed that the protein levels of IL- 6 and TNF- $\alpha$ dramatically increased in the surgery group compared with the control group; however, BMSC transplantation and TSG-6 injection significantly decreased the protein levels of IL- 6 and TNF- $\alpha$ compared with the surgery alone group (Fig. 7i, j).

\section{Discussion}

Cell-based therapy, such as BMSC transplantation, is a promising approach to halt and reverse the progress of IDD [44]. However, the potential mechanisms underlying the protective effects of BMSCs on the intervertebral discs are not completely understood. In the present study, we have found that BMSC treatment reduced the expression of MMPs, such as MMP-3 and MMP-13, and increased the expression of collagen II and aggrecan in IL-1 $\beta$-treated NPCs, possibly through enhanced expression of TSG-6; in turn, TSG-6 attenuated NPC degeneration by suppressing the activation of the TLR2/NF- $\mathrm{KB}$ signaling pathway. We also found that BMSCs and TSG-6 promote the proliferation of IL-1 $\beta$-treated NPCs. Furthermore, we have demonstrated that BMSC transplantation and TSG-6 injection enables the recovery of degenerated discs through inhibiting the TLR2/NF- $\kappa \mathrm{B}$ signaling pathway and decreasing the levels of NF-kB-dependent inflammatory cytokines and
MMPs in vivo. Taken together, the current research first demonstrated that TSG-6 secreted by BMSCs attenuates IDD via inhibition of the TLR2/NF- $\mathrm{KB}$ signaling pathway.

BMSCs are multipotential stem cells with relatively low immunogenicity, multipotency, and self-renewal capacity in vitro and in vivo. Recently, increasing studies have demonstrated that the protective effects of BMSCs might be due to their potent immunomodulatory and immunosuppressive properties [10-14]. Numerous studies have also found that BMSCs can inhibit inflammatory reactions and alter the host microenvironment by secreting a variety of bioactive molecules, such as anti-inflammatory proteins, growth factors, and trophic factors [45]. Recent research showed that paracrine factors secreted by BMSCs exert their anti-inflammation and anti-apoptotic effect on IDD through inhibiting the relative NF- $\mathrm{KB}$ and mitochondrial apoptotic pathways [46]. These data indicated that paracrine factors secreted by BMSCs play a protective role in IDD. TSG-6 is an anti-inflammatory protein that is expressed in many cell types in response to stimulation by several proinflammatory mediators $[47,48]$, such as TNF- $\alpha$. Several studies have found that TSG-6 secreted by BMSCs can produce anti-inflammatory effects in a wide variety of diseases, such as myocardial infarction, neurotraumatic injuries, retinal degeneration, sterile inflammation, colitis, and corneal epithelial wound [9, 37, 49-53]. In the present study, we showed that the expression of TSG-6 in BMSCs was upregulated significantly after stimulation by TNF- $\alpha$. In addition, after co-culture with activated BMSCs, the expression of MMP-3 and MMP-13 in IL-1 $\beta$-treated NPCs was significantly reduced; meanwhile, the expression of collagen II and aggrecan in IL- $1 \beta$-treated NPCs was significantly increased. However, when we used TSG-6 siRNA to knock down the expression of the TSG-6 gene in activated BMSCs, the protective effects of BMSCs on IL-1 $\beta$ treated NPCs compared with non-transfected BMSCs or NC siRNA-transfected BMSCs were partly weakened. To further confirm the key role of TSG-6 in ameliorating IL-1 $\beta$ induced NPC degeneration, we added recombinant TSG-6 protein directly to the NPC culture medium during IL-1 $\beta$ treatment. The recombinant TSG-6 protein also reduced the expression of MMP-3 and MMP-13 and increased the expression of collagen II and aggrecan in IL- $1 \beta$-treated NPCs in a dose-dependent manner. These results demonstrated that TSG- 6 made a major contribution to the BMSCmediated inhibition of IL- $1 \beta$-induced NPC degeneration.

Previous studies have demonstrated that TLR2 is expressed at a substantially elevated level in degenerated human NPCs [23, 26], which then triggers the MyD88dependent pathway and finally results in the activation of transcription factors, including NF- $\mathrm{\kappa B}$. Furthermore, it has been demonstrated that TSG- 6 secreted by BMSCs can inhibit the activation of the TLR2/NF- $\mathrm{KB}$ signaling pathway 


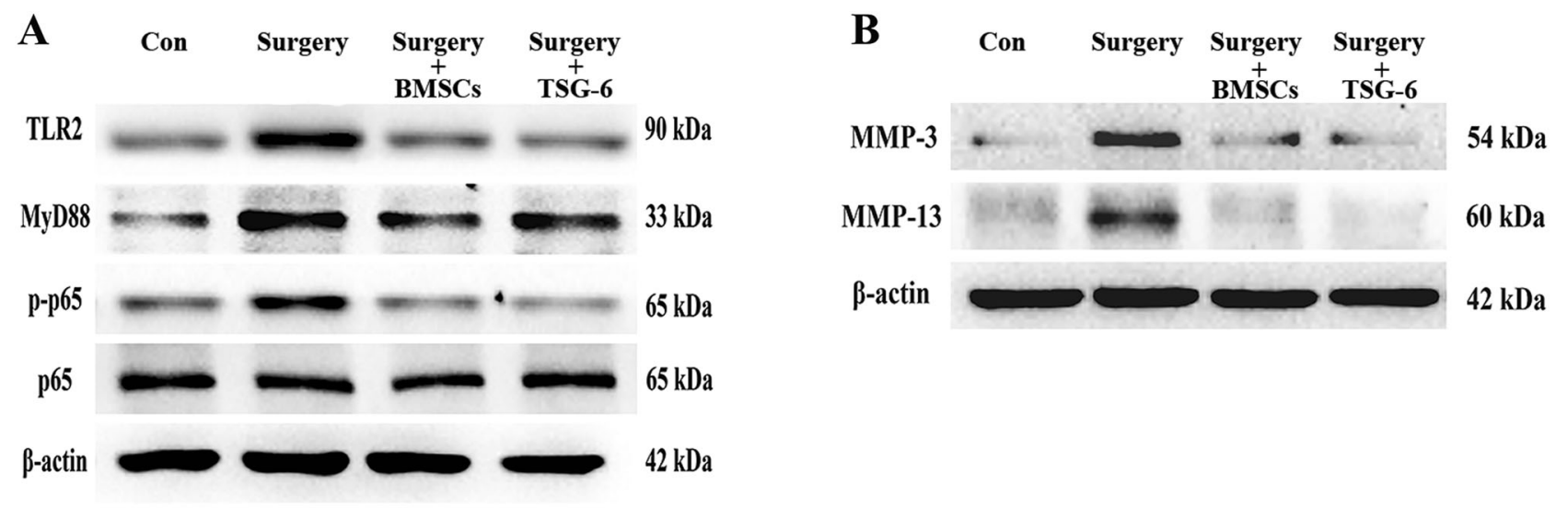

C

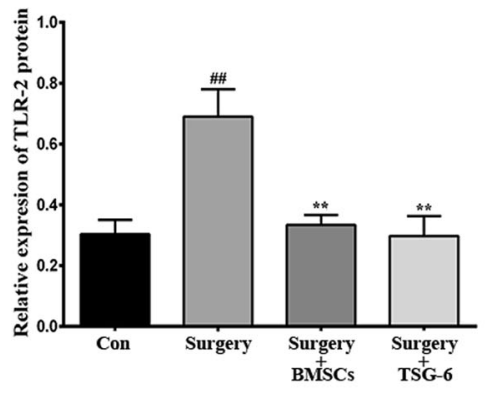

F

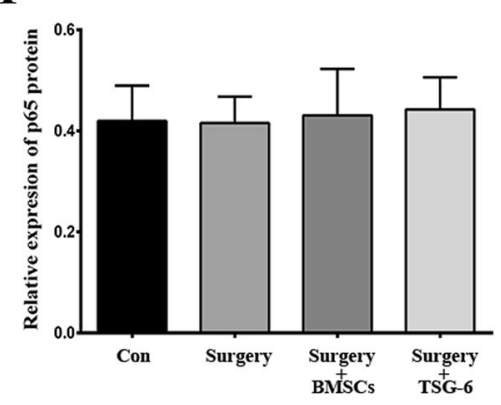

I

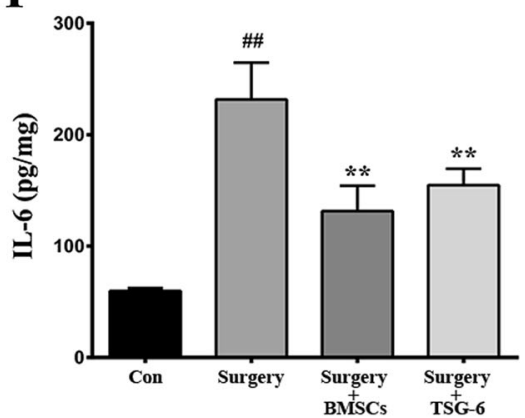

Fig. 7 BMSCs and TSG-6 downregulated the TLR2/NF- $\mathrm{BB}$ signaling pathway and reduced the protein levels of MMPs and inflammatory cytokines in degenerated NP tissue. The protein levels of TLR2, MyD88, p-p65, p65, MMP-3, and MMP-13 in NP tissue were measured by western blotting. a, b Representative image of protein levels of TLR2, MyD88, p-p65, p65, MMP-3, and MMP-13. c-h Quantitative analysis of western blotting results showed that BMSC

$\mathbf{J}$
$\mathbf{E}$

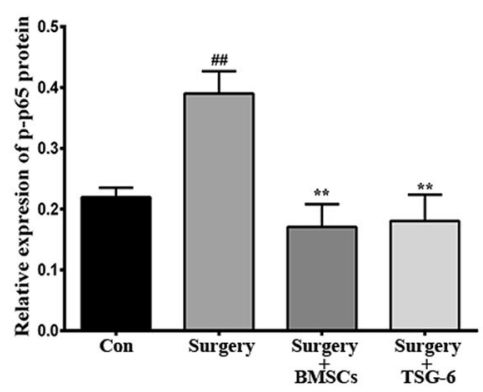

H

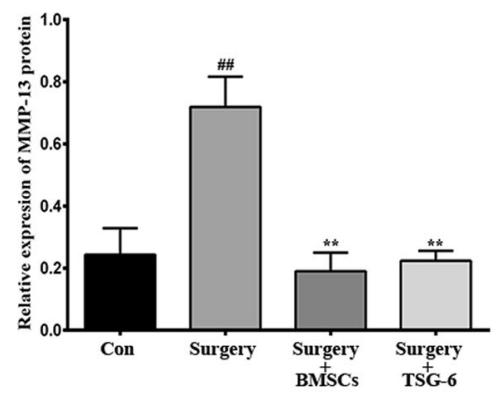

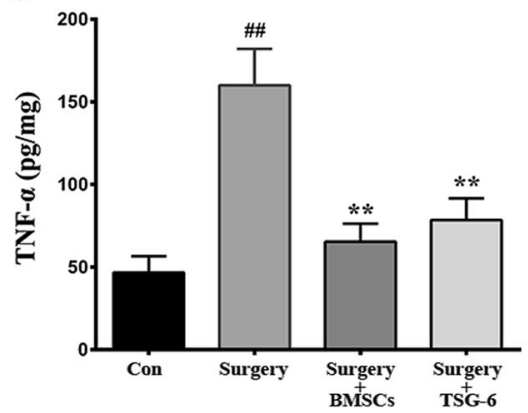

transplantation and TSG-6 injection significantly inhibited the activation of the TLR2/NF- $\mathrm{KB}$ signaling pathway and reduced the expression of MMPs in degenerated NP tissue. BMSC transplantation and TSG-6 injection significantly reduced the protein levels of $\mathbf{i}$ IL- 6 and $\mathbf{j}$ TNF- $\alpha$ in degenerated NP tissue. The data are expressed as the means $\pm \mathrm{SD}(n$ $=6$ in each group). ${ }^{\# \#} P<0.01$ versus the control group. ${ }^{* *} P<0.01$ versus the surgery group 
Fig. 8 Schematic of anti-IDD therapeutic effect of TSG-6 secreted by BMSCs. Proinflammatory cytokines, such as IL-1 $\beta$, activated NF- $\mathrm{KB}$ signaling in NPCs through TLR2. Activated NF- $\mathrm{\kappa B}$ increased the production of proinflammatory cytokines and MMPs. BMSCs were activated by the pro-inflammatory cytokines to secrete antiinflammatory protein TSG-6. TSG-6 secreted by BMSCs suppressed the TLR2/NF- $\mathrm{KB}$ signaling pathway in degenerated NPCs

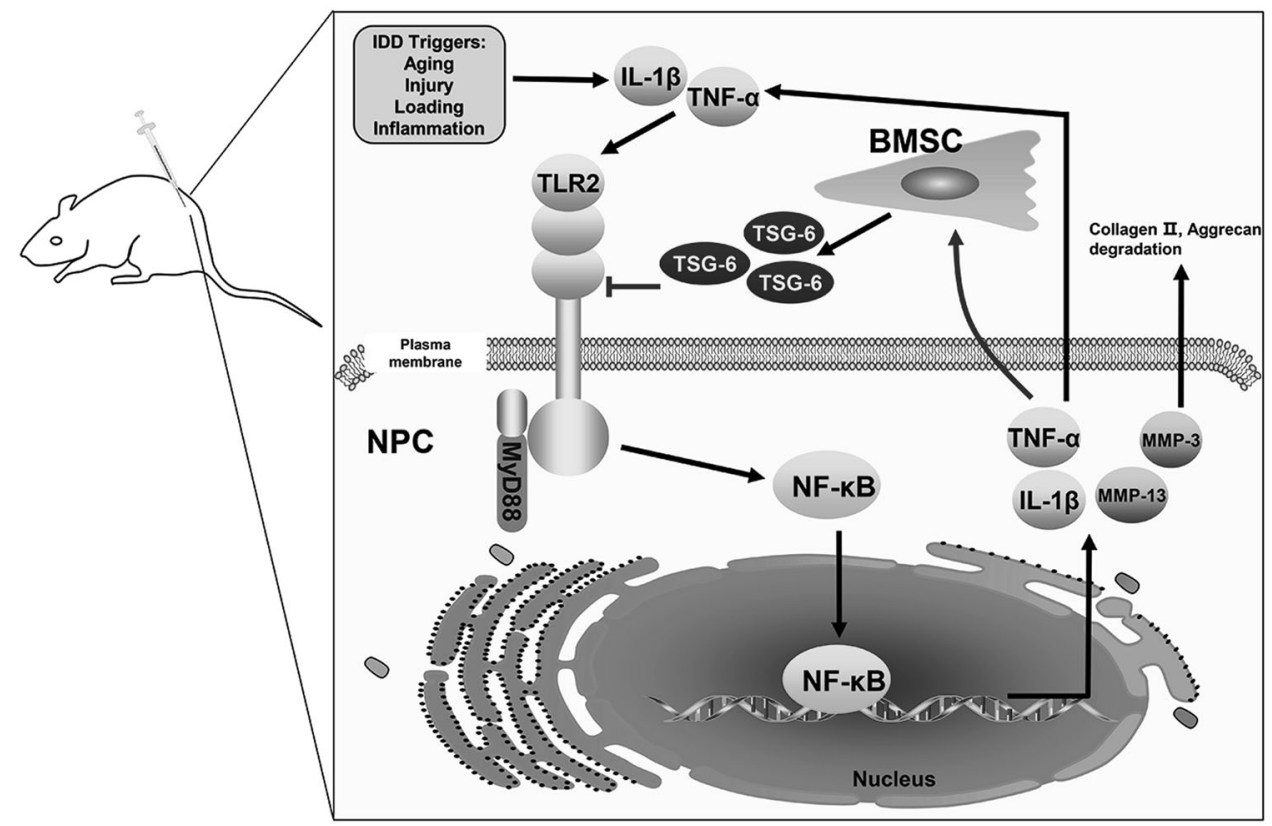

[36]. Consistent with previous observations, we found that the protein levels of TLR2 and MyD88 were increased in IL-1 $\beta$-treated NPCs and that the upregulation of TLR2 and MyD88 in IL-1 $\beta$-treated NPCs was inhibited after coculture with BMSCs or treatment with TSG-6.

$\mathrm{NF}-\kappa \mathrm{B}$ plays a crucial role in regulating the expression of a variety of pro-inflammatory cytokine genes. Under unstimulated conditions, NF- $\mathrm{kB}$ is localized in the cytoplasm and is bound to the inhibitory IкB protein, which prevents it from entering the nucleus. After stimulation, specific kinases phosphorylate IKB, causing its rapid degradation by proteasomes; IкB degradation allows NF- $\kappa \mathrm{B}$ to translocate into the nucleus, where it binds to specific sequences in the promoter regions of target genes [54, 55]. To elucidate the molecular mechanism underlying the antiinflammatory effects of TSG-6 in degenerated NPCs, we first examined the nuclear translocation of NF- $\mathrm{kB}$ p65. Our results showed that BMSC co-culture and TSG- 6 treatment substantially suppressed IL-1 $\beta$-induced nuclear translocation of NF-kB p65 in NPCs. Then, we evaluated the phosphorylation level of p65 by western blotting. Our data showed that an enhanced level of p65 phosphorylation was found in IL-1 $\beta$-treated NPCs, and the protein level of p-p65 in IL-1 $\beta$-treated NPCs was decreased after co-culture with BMSCs or treatment with TSG-6. Moreover, we used the specific TLR2 agonist Pam3CSK4 to further confirm that BMSCs and TSG-6 exert their beneficial effects on NPCs by suppressing the TLR2/NF- $\kappa B$ signaling pathway. Our results suggested that Pam3CSK4 treatment induced the protein expression of TLR2, MyD88, and p-p65 in NPCs; however, the expression of TLR2, MyD88, and p-p65 was inhibited after co-culture with BMSCs and treatment with
TSG-6 in Pam3CSK4-treated NPCs. The above-mentioned results demonstrated that TSG- 6 secreted by BMSCs exerts its protective effect partly through suppressing the activation of the TLR2/NF- $\mathrm{KB}$ signaling pathway to reduce NPC degeneration.

According to previous studies, a variety of proinflammatory cytokines, such as IL- $1 \alpha$, IL- $1 \beta$, IL- 6 , and TNF- $\alpha$, are significantly increased in degenerative intervertebral discs; $[56,57]$ these pro-inflammatory cytokines aggravate ECM catabolism and inflammation, ultimately resulting in structural and functional deterioration of the intervertebral discs [58]. Thus, inhibition of the production of pro-inflammatory cytokines can prevent or delay the degenerative process in intervertebral discs. In the present study, we used ELISA assays to investigate the effects of BMSCs and TSG-6 on pro-inflammatory cytokine secretion in degenerative NPCs. Our data showed that BMSCs and TSG-6 markedly inhibited IL-1 $\beta$ - or Pam3CSK4-induced IL- 6 and TNF- $\alpha$ secretion in NPCs. In addition, a recent study revealed that TSG-6 secreted by BMSCs can increase the proliferation of corneal epithelial cell and then repaired the corneal injury [53]. Consistent with previous studies, we also found that the proliferation of IL- $1 \beta$-treated NPCs significantly increased after co-culture with BMSCs or treatment with TSG-6.

Given that the in vitro experiment showed encouraging results, we further evaluated the protective value of BMSCs and TSG-6 on IDD in vivo, injecting BMSCs or TSG-6 into the NP tissue of a puncture-induced IDD model. Through histological analysis, we found that the structure of the intervertebral discs was well preserved after BMSC transplantation and TSG-6 injection: the NP consisted of 
numerous nuclear cells, the AF was arranged regularly, and the boundary between the NP and AF was minimally interrupted. In addition, Safranin O/Fast Green staining also showed that BMSC transplantation and TSG-6 injection significantly reduced the PG loss in intervertebral discs caused by needle puncture. MRI scanning showed that BMSC transplantation and TSG-6 injection increased the water content in the NP tissue compared with the surgery group (Fig. 8). Our results are consistent with those of previous studies, which also showed significantly elevated signal intensity and water content in NP tissue after transplantation of BMSCs [59, 60].

Collagen II and aggrecan are the two main components of NP tissue, and numerous studies have demonstrated that the reduction of collagen II and aggrecan is highly correlated with IDD[61, 62]. Previous studies have found that mesenchymal stem cell transplantation can upregulate the expression of collagen II and aggrecan [62, 63]. Consistent with these findings, after BMSC transplantation, the expression of collagen II and aggrecan in NP tissue was significantly greater than that observed in the surgery group. In addition, TSG-6 injection also increased the expression of collagen II and aggrecan. These data indicated that TSG6 secreted by BMSCs exerts its therapeutic effect by increasing the protein level of collagen II and aggrecan, resulting in maintenance of the intervertebral disc structure in vivo.

Our in vivo data also showed that BMSC transplantation and TSG-6 injection significantly inhibited the expression of TLR2, MyD88, p-p65, MMP-3, and MMP-13. MMPs play an important role in the pathophysiology of intervertebral disc disease and are believed to be the major matrix degrading enzymes in IDD [64]. These enzymes not only directly degrade the ECM, but are also responsible for the activation of other latent enzymes [65]. Various studies have found that the activated form of NF- $\mathrm{KB}$ mediated the expression of MMPs, such as MMP-3 and MMP-13, and accelerated degradation of the ECM in intervertebral disc [66, 67]. In the current study, an enhanced level of p65 phosphorylation was found in degenerated NP tissue. Meanwhile, the mRNA and protein levels of MMP-3 and MMP-13 were increased. In contrast, the expression of MMP-3 and MMP-13 was decreased when the activation of NF- $\mathrm{KB}$ was inhibited by BMSC transplantation and TSG-6 injection. Combined with these previous studies, our results indicated that the excessive activation of NF- $\mathrm{kB}$ lead to the upregulation of MMPs and subsequently degrade the ECM in degenerated intervertebral discs. Finally, our results showed that BMSC transplantation and TSG-6 injection significantly reduced inflammatory cytokines, such as IL- 6 and TNF- $\alpha$, in degenerated NP tissue. These data further confirmed that TSG-6 secreted by BMSCs exerts its therapeutic effect on degenerated NP tissue by suppressing the TLR2/NF- $\mathrm{BB}$ signaling pathway and reducing the production of inflammatory cytokines and MMPs.

It has been reported that the interaction of TSG- 6 with CD44 can decrease TLR2-mediated stimulation of NF- $\mathrm{KB}$ signaling $[36,68,69]$. CD44 is a transmembrane adhesion molecule and the major receptor for extracellular matrix proteins that are involved in cell migration, proliferation, and cell-cell interaction [70]. In the present study, we found that TSG-6 secreted by BMSCs significantly inhibited the activation of the TLR2/NF- $\mathrm{KB}$ signaling pathway in degenerated NPCs and NP tissue. Furthermore, previous study has proved that CD44 also expressed in the normal and IL-1 $\beta$-treated NPCs [71, 72]. Thus, we suggested that TSG- 6 negatively regulated the TLR2/NF$\kappa B$ signaling-mediated responses through $\mathrm{CD} 44$ in degenerated intervertebral discs. Further research needs to be performed to determine the specific mechanism between TSG-6 and TLR2/NF- $\mathrm{KB}$ signaling pathway in NPCs.

The present research confirmed that BMSCs inhibited the IL-1 $\beta$-induced activation of the TLR2/NF- $\mathrm{KB}$ signaling pathway and reduced the production of pro-inflammatory cytokines IL- 6 and TNF- $\alpha$ in NPCs by secreting the antiinflammatory protein TSG-6. In addition, our study demonstrated that BMSC transplantation could delay or even prevent disc degeneration partly through a TSG-6mediated anti-inflammatory effect in vivo.

Acknowledgements We thank Xiyao $\mathrm{Gu}$ and Zhongwei Yang for advice on the study design. We thank Xuemei Chen, Dan Tang, and Ryan Dowsell for helpful discussions. This work was supported by the National Natural Science Foundation of China (No. 81370178, No. 81570068).

\section{Compliance with ethical standards}

Conflict of interest The authors declare that they have no conflict of interest.

\section{References}

1. Meisel HJ. The lumbar vertebral disc. Eur Spine J. 2006;15(Suppl 3):S301-2.

2. Modic MT, Ross JS. Lumbar degenerative disk disease. Radiology. 2007;245:43-61.

3. Antoniou J, Steffen T, Nelson F, et al. The human lumbar intervertebral disc: evidence for changes in the biosynthesis and denaturation of the extracellular matrix with growth, maturation, ageing, and degeneration. J Clin Invest. 1996;98:996-1003.

4. Kraemer J. Natural course and prognosis of intervertebral disc diseases. International Society for the Study of the Lumbar Spine Seattle, Washington, 1994. Spine. 1995;20:635-9.

5. Levin DA, Hale JJ, Bendo JA. Adjacent segment degeneration following spinal fusion for degenerative disc disease. Bull NYU Hosp Jt Dis. 2007;65:29-36. 
6. Richardson SM, Mobasheri A, Freemont AJ, Hoyland JA. Intervertebral disc biology, degeneration and novel tissue engineering and regenerative medicine therapies. Histol Histopathol. 2007;22:1033-41.

7. Fleming JE Jr, Cornell CN, Muschler GF. Bone cells and matrices in orthopedic tissue engineering. Orthop Clin North Am. 2000;31:357-74.

8. Adams MA, Roughley PJ. What is intervertebral disc degeneration, and what causes it? Spine (Phila Pa 1976). 2006;31:2151-61.

9. Lee RH, Pulin AA, Seo MJ, et al. Intravenous hMSCs improve myocardial infarction in mice because cells embolized in lung are activated to secrete the anti-inflammatory protein TSG-6. Cell Stem Cell. 2009;5:54-63.

10. Prockop DJ. Repair of tissues by adult stem/progenitor cells (MSCs): controversies, myths, and changing paradigms. Mol Ther. 2009;17:939-46.

11. Nauta AJ, Fibbe WE. Hnmunomodulatory properties of mesenchymal stromal cells. Blood. 2007;110:3499-506.

12. Uccelli A, Moretta L, Pistoia V. Mesenchymal stem cells in health and disease. Nat Rev Immunol. 2008;8:726-36.

13. Caplan AI. Why are MSCs therapeutic? New data: new insight. J Pathol. 2009;217:318-24.

14. Ohtaki H, Ylostalo JH, Foraker JE, et al. Stem/progenitor cells from bone marrow decrease neuronal death in global ischemia by modulation of inflammatory/immune responses. Proc Natl Acad Sci USA. 2008;105:14638-43.

15. Yang $\mathrm{H}, \mathrm{Cao} \mathrm{C}, \mathrm{Wu} \mathrm{C}$, et al. TGF-betal suppresses inflammation in cell therapy for intervertebral disc degeneration. Sci Rep. 2015;5:13254.

16. Le Maitre CL, Pockert A, Buttle DJ, Freemont AJ, Hoyland JA. Matrix synthesis and degradation in human intervertebral disc degeneration. Biochem Soc Trans. 2007;35:652-5.

17. Le Maitre CL, Freemont AJ, Hoyland JA. The role of interleukin1 in the pathogenesis of human intervertebral disc degeneration. Arthritis Res Ther. 2005;7:R732-45.

18. Xu K, Chen W, Wang X, et al. Autophagy attenuates the catabolic effect during inflammatory conditions in nucleus pulposus cells, as sustained by NF-kappaB and JNK inhibition. Int J Mol Med. 2015;36:661-8

19. Ye W, Zhou J, Markova DZ, et al. Xylosyltransferase-1 expression is refractory to inhibition by the inflammatory cytokines tumor necrosis factor alpha and IL-1beta in nucleus pulposus cells: novel regulation by AP-1, Sp1, and Sp3. Am J Pathol. 2015;185:485-95.

20. Wang $\mathrm{X}, \mathrm{Li} \mathrm{H}, \mathrm{Xu} \mathrm{K}$, et al. SIRT1 expression is refractory to hypoxia and inflammatory cytokines in nucleus pulposus cells: Novel regulation by HIF-1alpha and NF-kappaB signaling. Cell Biol Int. 2016;40:716-26.

21. Lasker MV, Nair SK. Intracellular TLR signaling: a structural perspective on human disease. J Immunol. 2006;177:11-6.

22. Akira S, Uematsu S, Takeuchi O. Pathogen recognition and innate immunity. Cell. 2006;124:783-801.

23. Klawitter M, Hakozaki M, Kobayashi H, et al. Expression and regulation of toll-like receptors (TLRs) in human intervertebral disc cells. Eur Spine J. 2014;23:1878-91.

24. Quero L, Klawitter M, Schmaus A, et al. Hyaluronic acid fragments enhance the inflammatory and catabolic response in human intervertebral disc cells through modulation of toll-like receptor 2 signalling pathways. Arthritis Res Ther. 2013;15:R94.

25. Fang F, Jiang D. IL-1beta/HMGB1 signalling promotes the inflammatory cytokines release via TLR signalling in human intervertebral disc cells. Biosci Rep 2016;36. pii: e00379. https:// doi.org/10.1042/BSR20160118.

26. Shen J, Fang J, Hao J, et al. SIRT1 inhibits the catabolic effect of IL-1beta through TLR2/SIRT1/NF-kappaB pathway in human degenerative nucleus pulposus cells. Pain Physician. 2016;19: E215-26.

27. Wuertz K, Vo N, Kletsas D, Boos N. Inflammatory and catabolic signalling in intervertebral discs: the roles of NF-KB and MAP kinases. Eur Cell Mater. 2012;23:103-20.

28. Hayden MS, West AP, Ghosh S. NF-kappa B and the immune response. Oncogene. 2006;25:6758-80.

29. Furia B, Deng L, Wu K, et al. Enhancement of nuclear factorkappa B acetylation by coactivator p300 and HIV-1 Tat proteins. J Biol Chem. 2002;277:4973-80.

30. Adams MA, Stefanakis M, Dolan P. Healing of a painful intervertebral disc should not be confused with reversing disc degeneration: implications for physical therapies for discogenic back pain. Clin Biomech (Bristol, Avon). 2010;25:961-71.

31. Hoyland JA, Le Maitre C, Freemont AJ. Investigation of the role of IL-1 and TNF in matrix degradation in the intervertebral disc. Rheumatology (Oxf). 2008;47:809-14.

32. Ulrich JA, Liebenberg EC, Thuillier DU, Lotz JC. ISSLS prize winner: repeated disc injury causes persistent inflammation. Spine (Phila Pa 1976). 2007;32:2812-9.

33. Nerlich AG, Bachmeier BE, Schleicher E, et al. Immunomorphological analysis of RAGE receptor expression and NF-kappaB activation in tissue samples from normal and degenerated intervertebral discs of various ages. Ann N Y Acad Sci. 2007;1096:239-48.

34. Yao Z, Nie L, Zhao Y, et al. Salubrinal suppresses IL-17-induced upregulation of MMP-13 and extracellular matrix degradation through the NF-kB pathway in human nucleus pulposus cells. Inflammation. 2016;39:1997-2007.

35. Kang L, Hu J, Weng Y, Jia J, Zhang Y. Sirtuin 6 prevents matrix degradation through inhibition of the NF-kappaB pathway in intervertebral disc degeneration. Exp Cell Res. 2017;352:322-32.

36. Choi H, Lee RH, Bazhanov N, Oh JY, Prockop DJ. Antiinflammatory protein TSG-6 secreted by activated MSCs attenuates zymosan-induced mouse peritonitis by decreasing TLR2/NFkappaB signaling in resident macrophages. Blood. 2011;118:330-8.

37. Liu Y, Zhang R, Yan K, et al. Mesenchymal stem cells inhibit lipopolysaccharide-induced inflammatory responses of BV2 microglial cells through TSG-6. J Neuroinflamm. 2014;11:135.

38. Krock E, Rosenzweig DH, Currie JB, et al. Toll-like receptor activation induces degeneration of human intervertebral discs. Sci Rep. 2017;7:17184.

39. Krock E, Currie JB, Weber MH, et al. Nerve growth factor is regulated by Toll-like receptor 2 in human intervertebral discs. $\mathrm{J}$ Biol Chem. 2016;291:3541-51.

40. Roughley PJ. Biology of intervertebral disc aging and degeneration: involvement of the extracellular matrix. Spine (Phila $\mathrm{Pa}$ 1976). 2004;29:2691-9.

41. Sive JI, Baird P, Jeziorsk M, et al. Expression of chondrocyte markers by cells of normal and degenerate intervertebral discs. Mol Pathol. 2002;55:91-7.

42. Sivan SS, Wachtel E, Roughley P. Structure, function, aging and turnover of aggrecan in the intervertebral disc. Biochim Biophys Acta. 2014;1840:3181-9.

43. Kluba T, Niemeyer T, Gaissmaier C, Grunder T. Human anulus fibrosis and nucleus pulposus cells of the intervertebral disc: effect of degeneration and culture system on cell phenotype. Spine (Phila Pa 1976). 2005;30:2743-8.

44. Fassett DR, Kurd MF, Vaccaro AR. Biologic solutions for degenerative disk disease. J Spinal Disord Tech. 2009;22:297-308.

45. Meirelles Lda S, Fontes AM, Covas DT, Caplan AI. Mechanisms involved in the therapeutic properties of mesenchymal stem cells. Cytokine Growth Factor Rev. 2009;20:419-27. 
46. Hu J, Yan Q, Shi C, et al. BMSC paracrine activity attenuates interleukin-1beta-induced inflammation and apoptosis in rat $\mathrm{AF}$ cells via inhibiting relative NF-kappaB signaling and the mitochondrial pathway. Am J Transl Res. 2017;9:79-89.

47. Milner CM, Day AJ. TSG-6: a multifunctional protein associated with inflammation. J Cell Sci. 2003;116:1863-73.

48. Wisniewski HG, Vilcek J. TSG-6: an IL-1/TNF-inducible protein with anti-inflammatory activity. Cytokine Growth Factor Rev. 1997;8:143-56.

49. Tuo J, Cao X, Shen D, et al. Anti-inflammatory recombinant TSG6 stabilizes the progression of focal retinal degeneration in a murine model. J Neuroinflamm. 2012;9:59.

50. Zhang R, Liu Y, Yan K, et al. Anti-inflammatory and immunomodulatory mechanisms of mesenchymal stem cell transplantation in experimental traumatic brain injury. $\mathrm{J}$ Neuroinflamm. 2013;10:106

51. Lee RH, Yu JM, Foskett AM, et al. TSG-6 as a biomarker to predict efficacy of human mesenchymal stem/progenitor cells (hMSCs) in modulating sterile inflammation in vivo. Proc Natl Acad Sci USA. 2014;111:16766-71.

52. Sala E, Genua M, Petti L, et al. Mesenchymal stem cells reduce colitis in mice via release of TSG6, independently of their localization to the intestine. Gastroenterology. 2015;149:163-76.e20.

53. Di G, Du X, Qi X, et al. Mesenchymal stem cells promote diabetic corneal epithelial wound healing through TSG-6-dependent stem cell activation and macrophage switch. Invest Ophthalmol Vis Sci. 2017;58:4344-54.

54. Barnes PJ, Karin M. Nuclear factor-kappaB: a pivotal transcription factor in chronic inflammatory diseases. $\mathrm{N}$ Engl J Med. 1997;336:1066-71.

55. Chen ZJ, Parent L, Maniatis T. Site-specific phosphorylation of IkappaBalpha by a novel ubiquitination-dependent protein kinase activity. Cell. 1996;84:853-62.

56. Lee S, Moon CS, Sul D, et al. Comparison of growth factor and cytokine expression in patients with degenerated disc disease and herniated nucleus pulposus. Clin Biochem. 2009;42:1504-11.

57. Sainoh T, Orita S, Miyagi M, et al. Interleukin-6 and interleukin-6 receptor expression, localization, and involvement in pain-sensing neuron activation in a mouse intervertebral disc injury model. J Orthop Res. 2015;33:1508-14.

58. Risbud MV, Shapiro IM. Role of cytokines in intervertebral disc degeneration: pain and disc content. Nat Rev Rheumatol. 2014;10:44-56.

59. Sun W, Zhang K, Liu G, et al. Sox9 gene transfer enhanced regenerative effect of bone marrow mesenchymal stem cells on the degenerated intervertebral disc in a rabbit model. PLoS One. 2014;9:e93570
60. Henriksson HB, Svanvik T, Jonsson M, et al. Transplantation of human mesenchymal stems cells into intervertebral discs in a xenogeneic porcine model. Spine. 2009;34:141-8.

61. Tu J, Li W, Zhang Y, et al. Simvastatin inhibits IL-1beta-induced apoptosis and extracellular matrix degradation by suppressing the NF-kB and MAPK pathways in nucleus pulposus cells. Inflammation. 2017;40:725-34.

62. Pereira CL, Teixeira GQ, Ribeiro-Machado C, et al. Mesenchymal stem/stromal cells seeded on cartilaginous endplates promote intervertebral disc regeneration through extracellular matrix remodeling. Sci Rep. 2016;6:33836.

63. Cai F, Wu XT, Xie XH, et al. Evaluation of intervertebral disc regeneration with implantation of bone marrow mesenchymal stem cells (BMSCs) using quantitative T2 mapping: a study in rabbits. Int Orthop. 2015;39:149-59.

64. Wuertz K, Vo N, Kletsas D, Boos N. Inflammatory and catabolic signalling in intervertebral discs: the roles of NF-kappaB and MAP kinases. Eur Cell Mater. 2012;23:103-19. discussion19-20

65. Kepler CK, Ponnappan RK, Tannoury CA, Risbud MV, Anderson DG. The molecular basis of intervertebral disc degeneration. Spine J. 2013;13:318-30.

66. Yin J, Ren K, Huang Y, et al. Exploration about changes of IL-10, NF-kappaB and MMP-3 in a rat model of cervical spondylosis. Mol Immunol. 2018;93:184-8.

67. Wang S, Liu C, Sun Z, et al. IL-1beta increases asporin expression via the NF-kappaB p65 pathway in nucleus pulposus cells during intervertebral disc degeneration. Sci Rep. 2017;7:4112.

68. Lesley J, Gal I, Mahoney DJ, et al. TSG-6 modulates the interaction between hyaluronan and cell surface CD44. J Biol Chem. 2004;279:25745-54.

69. Kota DJ, Wiggins LL, Yoon N, Lee RH. TSG-6 produced by hMSCs delays the onset of autoimmune diabetes by suppressing Th1 development and enhancing tolerogenicity. Diabetes. 2013;62:2048-58

70. Ilangumaran S, Borisch B, Hoessli DC. Signal transduction via CD44: role of plasma membrane microdomains. Leuk Lymphoma. 1999;35:455-69.

71. Isa IL, Srivastava A, Tiernan D, et al. Hyaluronic acid based hydrogels attenuate inflammatory receptors and neurotrophins in interleukin-1beta induced inflammation model of nucleus pulposus cells. Biomacromolecules. 2015;16:1714-25.

72. Erwin WM, Islam D, Inman RD, Fehlings MG, Tsui FW. Notochordal cells protect nucleus pulposus cells from degradation and apoptosis: implications for the mechanisms of intervertebral disc degeneration. Arthritis Res Ther. 2011;13:R215. 